Chapter 19

\title{
Possibility of RFID in Conditions of Postal Operators
}

\author{
Juraj Vaculík, Peter Kolarovszki and Jiří Tengler \\ Additional information is available at the end of the chapter \\ http://dx.doi.org/10.5772/53285
}

\section{Introduction}

Radio frequency identification is becoming a modern trend in many sectors. It provides a contactless identification, tracking and tracing of goods, property and people in real time. Increase efficiency, performance and competitiveness. One area of application of RFID technology is also postal processes. In this context there are several question of feasibility of the use of identification of letters. In addition to the costs associated with the introduction of technology is necessary to examine the feasibility of using RFID technology in the field of postal processes.

Today, postal operations have implemented RFID in various closed-loop systems to measure, monitor, and improve operations. For example, RFID is being used to monitor international mail service between major hubs. By randomly "seeding" tagged letters into trays, elapsed delivery time can be measured. This allows service issues to be identified and addressed in a reliable and cost-effective manner.

Other postal operations have piloted tracking mail containers to measure trailer utilization and to track container locations. Manual container tracking systems tend to break down when volumes are high and there's a deadline to meet departure times. By allowing information to be captured automatically, RFID makes sure it is done, even under stressful conditions. Postal managers can rely on the information to make decisions that improve transportation costs and to relocate containers when needed. RFID-tracked mailbags, which provide delivery status, have already been created for priority mail services. Tagged mailbags are automatically read at specific points in the network to provide this automated track-and-trace capability. Four additional areas can benefit from the cheap, accurate, and pervasive information obtained using RFID. Each of them has the prospect for returning substantial monetary benefits, as well as having the potential to significantly upgrade postal service capabilities, an ever more important consideration in the competitive delivery market. 
Chapter is divided on several parts. We will be talk about basic of RFID, possibility of technology in postal and logistics processes, other mobile technology in processes, security of technology with contents to postal services, impact of operational characteristic on the readability and finally results of testing RFID technology in our laboratory of Automated identification and data capture (AIDC Lab) of University of Žilina.

\section{Basic of RFID technology architecture}

The RFID system architecture consists of a reader and a tag (also known as a label or chip). The reader queries the tag, obtains information, and then takes action based on that information. That action may display a number on a hand held device, or it may pass information on to a POS system, an inventory database or relay it to a backend payment system thousands of miles away. Let's looks at some of the basic components of a typical RFID system.

\subsection{RFID tag/label}

RFID units are in a class of radio devices known as transponders. A transponder is a combination transmitter and receiver, which is designed to receive a specific radio signal and automatically transmit a reply. Transponders used in RFID are commonly called tags, chips, or labels, which are fairly interchangeable, although "chip" implies a smaller unit, and "tag" is used for larger devices. The designator label is mainly used for the labels that contain an RFID device. Tags are categorized into four types based on the power source for communication and other functionality (Figure 1):

- A passive tag uses the electromagnetic energy it receives from an interrogator's transmission to reply to the interrogator. The reply signal from a passive tag, which is also known as the backscattered signal, has only a fraction of the power of the interrogator's signal. This limited power significantly restricts the operating range of the tag. Since passive tags are low power devices, they can only support data processing of limited complexity. On the other hand, passive tags typically are cheaper, smaller, and lighter than other types of tags, which are compelling advantages for many RFID applications. [3]

- An active tag relies on an internal battery for power. The battery is used to communicate to the interrogator, to power on-board circuitry, and to perform other functions. Active tags can communicate over greater distance than other types of tags, but they have a finite battery life and are generally larger and more expensive. Since these tags have internal power, they can respond to lower power signals than passive tags. [3]

- A semi-active tag is an active tag that remains dormant until it receives a signal from the interrogator to wake up. The tag can then use its battery to communicate with the interrogator. Like active tags, semi- active tags can communicate over a longer distance than passive tags. Their main advantage relative to active tags is that they have a longer battery life. The waking process, however, sometimes causes an unacceptable time delay when tags pass interrogators very quickly or when many tags need to be read within a very short period of time. [3] 
- A semi-passive tag is a passive tag that uses a battery to power on-board circuitry, but not to produce return signals. When the battery is used to power a sensor, they are often called sensor tags. They typically are smaller and cheaper than active tags, but have greater functionality than passive tags because more power is available for other purposes. Some literature uses the terms "semi-passive" and "semi- active" interchangeably. [3]

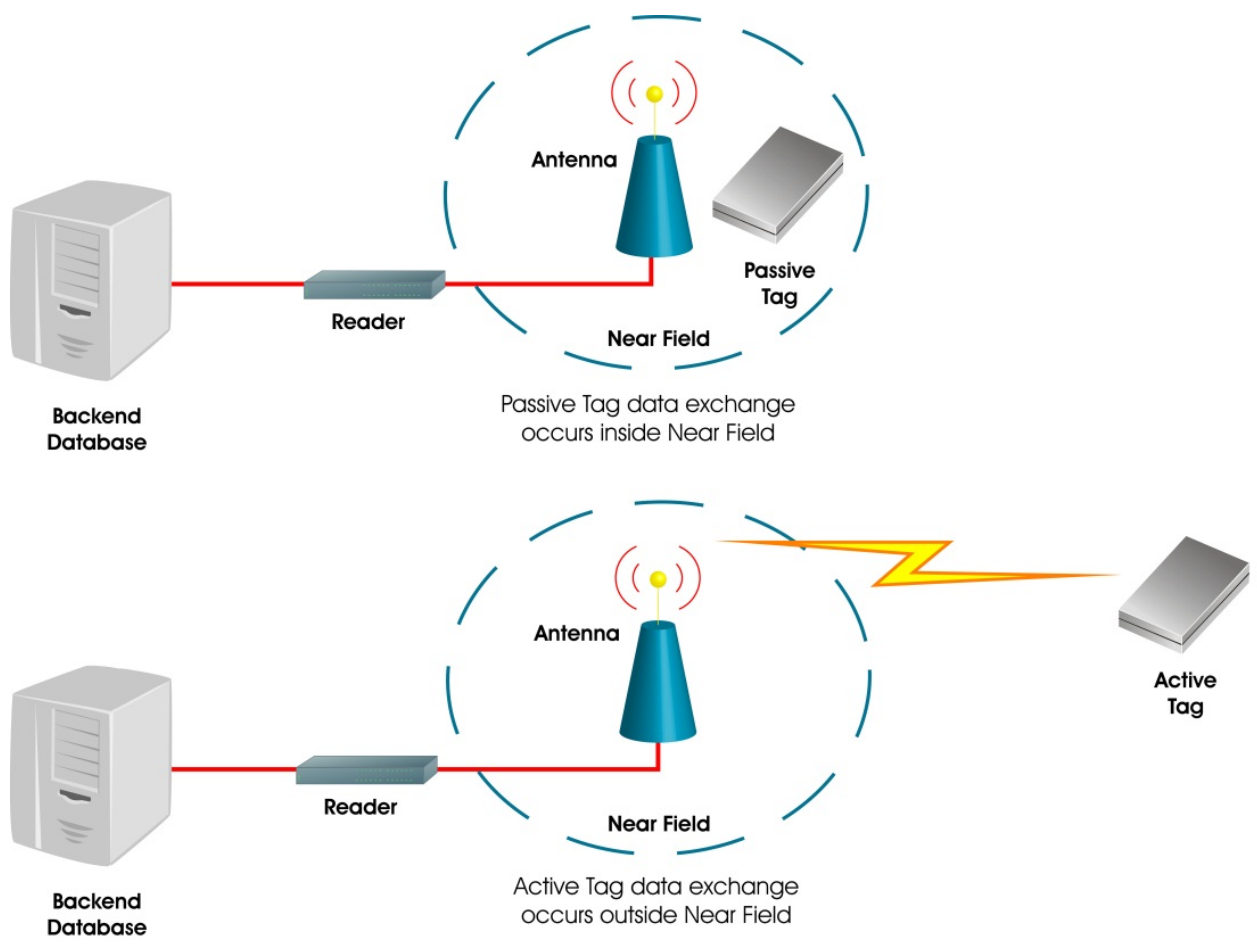

Figure 1. Passive and Active Tag Processes

Like bar codes in an earlier time, RFID is the next revolution in AIDC technology. Most of the advantages of RFID are derived from the reliance on radio frequencies rather than light (as is required in optical technology) to transmit information. This characteristic means that RFID communication can occur:

- Without optical line of sight, because radio waves can penetrate many opaque materials,

- At greater speeds, because many tags can be read quickly, whereas optical technology often requires time to manually reposition objects to make their bar codes visible, and

- Over greater distances, because many radio technologies can transmit and receive signals more effectively than optical technology under most operating conditions. [3] 


\subsubsection{Carrier frequencies}

Today, there are four carrier frequencies implemented for RFID that are popular globally: $125 \mathrm{KHz}, 13.56 \mathrm{MHz}$, UHF ranging from 866 to $950 \mathrm{MHz}$ depending on national radio regulations, and microwave frequencies of $2.45 \mathrm{GHz}$ and $5.8 \mathrm{GHz}$. There is also the frequency range $430-440 \mathrm{MHz}$, which is allocated to amateur radio usage around the world. The ISM band 433.05-434.790 MHz is located near the middle of the amateur radio band. The amateur radio band has emerged as an RFID channel in a number of applications. The frequency range has been called the 'optimal frequency for global use of Active RFID'. [1]

\subsubsection{Functionality}

- The primary function of a tag is to provide an identifier to an interrogator, but many types of tags support additional capabilities that are valuable for certain business processes. These include:

- Memory - memory enables data to be stored on tags and retrieved at a later time. Memory is either write once, read many (WORM) memory or re-writeable memory, which can be modified after initialization. Memory enables more flexibility in the design of RFID systems because RFID data transactions can occur without concurrent access to data stored in an enterprise subsystem. However, adding memory to a tag increases its cost and power requirements.

- Environmental sensors. The integration of environmental sensors with tags is an example of the benefit of local memory. The sensors can record temperature, humidity, vibration, or other phenomena to the tag's memory, which can later be retrieved by an interrogator. The integration of sensors significantly increases the cost and complexity of the tags. Moreover, while many tag operations can be powered using the electromagnetic energy from an interrogator, this approach is not workable for sensors, which must rely on battery power. Tags typically are only integrated with sensors for high-value, environmentally sensitive, or perishable objects worthy of the additional expense.

- Security functionality, such as password protection and cryptography. Tags with onboard memory are often coupled with security mechanisms to protect the data stored in that memory. For example, some tags support a lock command that, depending on its implementation, can prevent further modification of data in the tag's memory or can prevent access to data in the tag's memory. In some cases, the lock command is permanent and in other cases, an interrogator can "unlock" the memory. EPCglobal standards, International Organization for Standardization (ISO) standards, and many proprietary tag designs support this feature. Some RFID systems support advanced cryptographic algorithms that enable authentication mechanisms and data confidentiality features, although these functions are most commonly found on RFID-based contactless smart cards and not tags used for item management. Some tags offer tamper protection as a physical security feature.

- Privacy protection mechanisms. EPC tags support a feature called the kill command that permanently disables the ability of the tag to respond to subsequent commands. Unlike 
the lock command, the kill command is irreversible. The kill command also prevents access to a tag's identifier, in addition to any memory that may be on the tag. While the lock command provides security, the primary objective of the kill command is personal privacy. RFID tags could be used to track individuals that carry tagged items or wear tagged articles of clothing when the tags are no longer required for their intended use, such as to expedite checkout or inventory. The ability to disable a tag with the kill command provides a mechanism to prevent such tracking.[1]

\subsection{RFID reader (Interrogator)}

The second component in a basic RFID system is the interrogator or reader, which wirelessly communicate with a tag. Readers can have an integrated antenna, or the antenna can be separate. The antenna can be an integral part of the reader, or it can be a separate device. Handheld units are a combination reader/antenna, while larger systems usually separate the antennas from the reader.

The reader retrieves the information from the RFID tag. The reader may be self-contained and record the information internally; however, it may also be part of a localized system such as a POS cash register, a large Local Area Network (LAN), or a Wide Area Network (WAN).

There is also Middleware, software that controls the reader and the data coming from the tags and moves them to other database systems. It carries out basic functions, such as filtering, integration and control of the reader. [1]

RFID systems work, if the reader antenna transmits radio signals. These signals are captured tag, which corresponds to the corresponding radio signal (Figure 2).

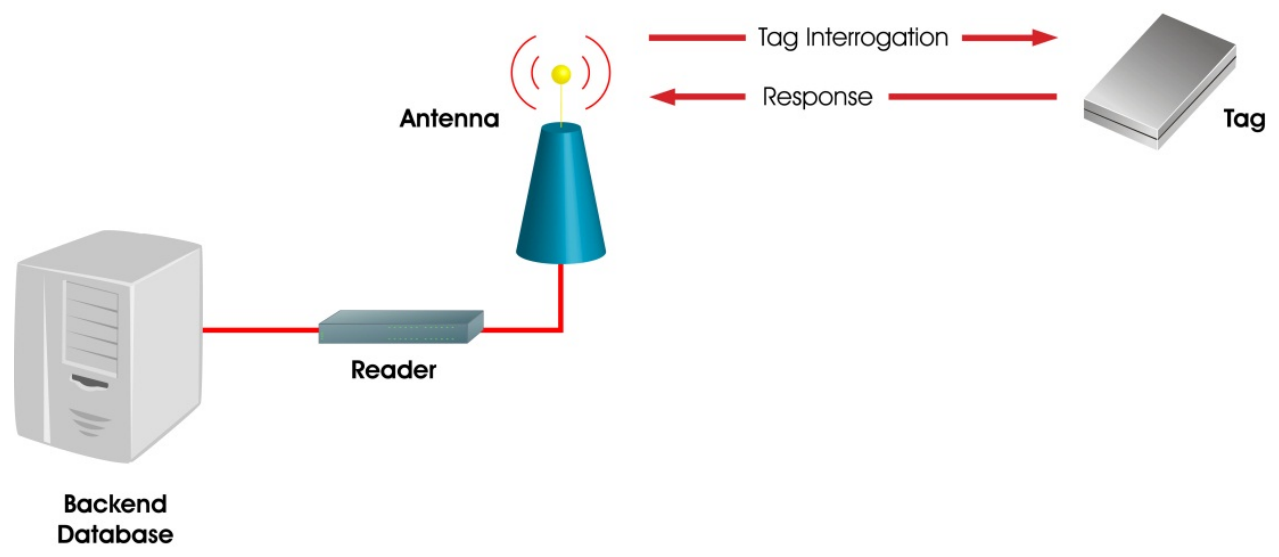

Figure 2. The interaction between the reader and RFID tag [2] 


\subsection{Security of RFID technology}

Let's start with the first question: What are the security risks with RFID? The information inside [passive] RFID tags is vulnerable to alteration, corruption, and deletion due to low processing speed and low memory. In contrast, some high-end active RFID readers and tags tend to improve security through use of cryptography, challenge-response protocols, rotating passwords, and tamper detection technology. These devices have more processing power and more memory than their passive counterparts. They are more expensive and need a battery to give a boost to the processing power. The passive RFID devices do not need a battery. The tags wake up when they receive a signal from a reader.

Now let's go the second question: How can we categorize the attacks on RFID technology? The management can start with the four categories of the attacks that are unique to the RFID infrastructure: war-walking and lifting, counterfeiting, denial-of-service, and weak cryptography.

\subsubsection{War-walking and war-lifting}

War-driving, also known as the wireless LAN driving is a technique of using a Wi-Fibased laptop or PDA to detect Wi-Fi wireless networks while driving in a vehicle, such as a small truck or an automobile. Legitimate war-drivers do not use services without proper authorization.

In the RFID technology arena, we add the wireless RFID driving to the description of wardriving. It is not necessary to have a LAN as an access point that a remote wireless device can pick up. A war-driver can use the device to pick up the information from unsecured tags affixed to an item, case, or pallet. What is more is that the war-driver could disable the RFID deactivation mechanisms when the items leave the retail stores.

In addition, the war-driver can read and get the information from the RFID tags of purchased goods that a passerby carries in a shopping bag. This can happen only if the tags are not properly deactivated when they leave a retail store or a warehouse.

War-walking is more bold than war-driving. War-walkers do not need a wireless device to find the RFID tags. With fake credentials or cards, they can bypass physical checks and find the system that uses RFID tags to monitor the movements of conference attendees.

Let's assume the cracker goes beyond finding the system. The cracker either runs away or removes the passive RFID tags from the objects, say, inside one case by sawing or etching the tags away. The cracker replaces them with the counterfeited tags, and reattaches the tag with original RFID data to the like objects in another case, all without being detected. This technique is known as lifting.

In another instance, a corporate spy walks around, scans the entire stock of a competing retail outlet, rewrites the tags of cheap products and replaces with better product labels and even hides products in a metal-lined tag and replaces with new tags on shelf. Passive tags do not work very well when they come into contact with a metallic surface. 
Another privacy issue that has raised is what flashes up on a scanner as someone walks near the interrogator (especially the active interrogators that have a much wider scanning region than those of passive interrogators). The scanner could show:

- Clothing origins

- Contents of origins

- Contents of briefcase or handbag

- Which credit cards being carried

- Linkage to RFIDs that identify the user of passport in suit pocket

Make sure the RFID infrastructure is secured with physical security control mechanisms. If the company can afford it, it could use, for example, AXCESS's ActiveTag system, a singlesystem approach to automatic monitoring and tracking applications right from your desktop computer, including Asset Management, Personnel and Vehicle Access Control, Personnel Monitoring, Production and Process Control, and Inventory Tracking.

\subsubsection{Counterfeiting}

It is the semi-conductor companies who manufacture RFID tags. Unlike security firms, the semi-conductors have practically no experience in security. These companies are more interested in getting the customers to buy their products rather than in the discussion of product vulnerabilities and countermeasures. Another problem is the vendors who become too overconfident that their products will not be easy to break.

With a switched reader, you will be not able to read the tags. An adversary can defeat an encryption by switching readers after gaining physical access to the location that sends encrypted communications.

Now, how does an adversary make the switch? One possibility is to switch with a fake reader. Another possibility is to tamper with the original reader. It is so easy to do so with a portable handheld device, particularly the ones that can fit into the palm of most hands. The tampered or replaced reader can be modified to allow the adversary to control a legitimate reader nearby from a distance and write counterfeit serial numbers on the RFID tags. It also can be modified to automatically change the original RFID numbers stored in the reader's database and replace it with invalid numbers.

That is why it is important to secure custody for the reader even when a RFID handler is not using the device. It is also important for the organizations to ensure that a legitimate reader can reject an invalid RFID number counterfeited on the tag or in the reader's database.

You should determine what countermeasures you need to mitigate the risks of counterfeiting threats before RFID is fully implemented. 


\subsubsection{Denial of service}

RFID radio signals area also very easy to block or jam. This can cause denial-of-service not only to the RFID tags but also at the data and network level.

Hackers and crackers can launch a denial-of-service attack by using electromagnetic fog to block RFID scanning and flooding a retail outlet with radio waves at the same frequencies as RFID scanners, thus causing chaos at check-outs. They also can hide a transmitter in a cat at a parking lot. This transmitter can block radio signals, causing an RFID-enabled store to close, and send a malicious virus to an EPC IS server containing the RFID data.

\subsubsection{Weak cryptography}

Although we expect the price for passive tags to drop below five cents per unit in a few years, we must acknowledge that these tags are computationally weak for the standard basic symmetric key cryptographic operations. Because more expensive RFID tags have more processing power and memory they can perform advanced cryptographic functions. Most low-cost tags are readable; many have limited writeable capability. This is because these tags are designed with basic functionality to keep the costs low.

Although we can get around this problem in a limited way via minimalist cryptography and Elliptic Curve Cryptography (ECC), they are more appropriate for other RFID devices, smart cards.

To overcome some of the confusing policies on when to use the kill command, the AUTO-ID Center and EPCglobal have proposed to put thef chip tags to sleep for a while rendering them inoperable temporarily and: then wake up these tags later on with a pair of sleep/wake commands.

As mentioned previously, the basic functionality of the low-cost RFID tags does not allow the basic cryptographic operations, due to limited processing power and little memory and size of the chip. To make it work, the tag must have memory of several megabytes and be rateable. The scheme for this cryptography is pseudonym throttling. It sores a short list of random identifiers of pseudonyms and goes into a cycle. Very little computation, if any, is involved, as contrasted to standard cryptography that requires quite a bit of computation and more complex circuitry.

The ECC is widely accepted for its efficient deployment of the public key mechanism. ECC is known for its compactness due to the novel way it uses arithmetic units to perform complex computations. It is much more compact then RSA, allowing the low-cost tags to be RFID-enabled. To get the ECC to work properly in RFID tags, we cannot overlook three important things: an adequate memory, the size of the area into which the ECC is installed, and the amount of power the tag can consume and emit signals to perform a simple computation. If the memory is too low, the ECC will not work. If the memory is adequate but the circuitry does not give enough power to consume, the ECC will not work. If the size of the area is too small regardless of memory size or the amount of power consumption, the ECC 
will not work. The memory, the area size, and power consumption, must be set properly in order for all three to get the computation to work properly.

\subsubsection{Defence in depth}

Let's assume light-weight cryptography for the RFID tag is well designed and is one of the protection mechanisms to defend the RFID infrastructure from attacks. In reality, 100 percent protection from cryptography is not possible. What is possible is the mitigation of risks to cryptographic attacks to an acceptable level. Another possibility is to let other protection mechanisms take over at the software/hardware level if one protection mechanism degrades or fails. They include firewalls, intrusion detection systems, scanners, RFID monitoring, failover servers, VPNs, and PKI.

As shown in Figure 3., these protections form the core of the Defense-in-Depth model of three rings. The middle ring focuses on access and audit controls. Access controls are best achieved with a WSSO for each user via SAML Auditing is accomplished with an examination of security practices and mechanisms within the organization.

Overlapping the core and middle rings are the operating systems that include both, for example, firewalls and access controls, such as Windows 2000 security, Windows 2003 Server Security, UNIX and Linux security, and Web security. Also included are the automated tools and devices to assess network vulnerabilities.

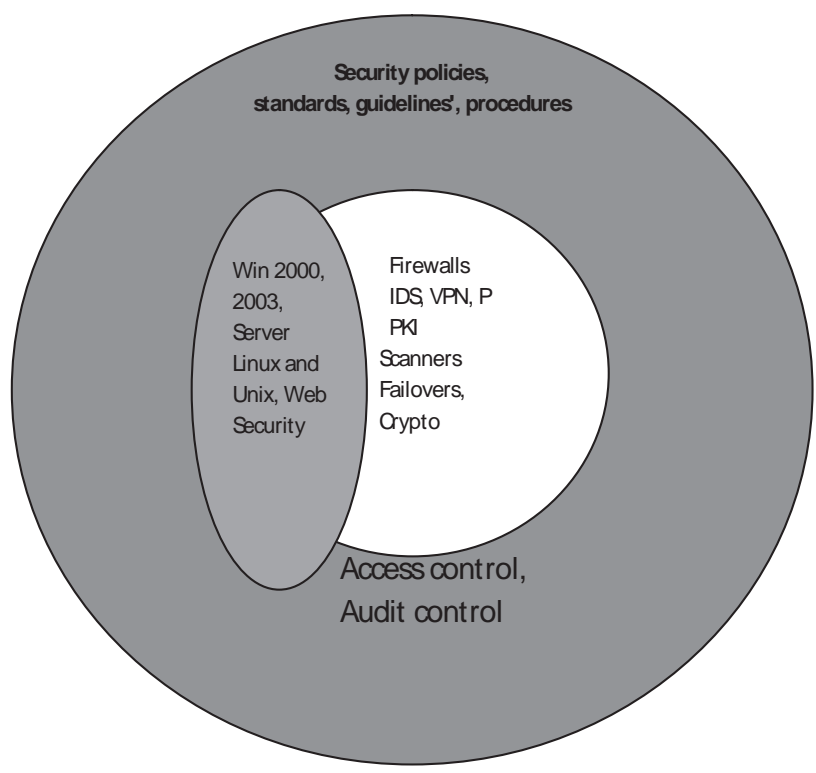

Figure 3. RFID Defence-In-Depth 
The outer ring is a set of security policies including business continuity policy, risk assessment policy, password protection management policy, and server security policy.

Implementing the Defense-in-Depth is not as easy as it seems. Administrators must often choose from among a dizzying array of specialized hardware and software products to meet their organizations' need for network security.

To realize both best-of-breed application choice and full management integration, network administrators should consider an enterprise security solution built on an open architectural platform. With well-defined interfaces, this enables third-party security applications to plug in seamlessly with the overall security policy. In addition, an open architecture can leverage Application Programming Interfaces (APIs) to develop and deploy custom applications to meet specific network security needs.

\subsection{RFID data collection tool-backend communication attack}

Middleware and backend communication occur using JMS, SOAP, or HTTP. There are two types of attacks that can have an impact on the backend: MIM application layer attack and a TCP replay attack.

\subsubsection{MIM Attack}

A MIM attack occurs when someone monitors the system between you and the person you are communicating with. When computers communicate at low levels of the network layer, they may not be able to determine who they are exchanging data with. In MIM attacks, someone assumes a user's identity in order to read his or her messages. The attacker might be actively replying as you to keep the exchange going and to gain more information. MIM attacks are more likely when there is less physical control of the network (e.g., over the Internet or over a wireless connection).

\subsubsection{Application layer attack}

An application layer attack targets application servers by deliberately causing a fault in a server's operating system or applications, which results in the attacker gaining the ability to bypass normal access controls. The attacker takes advantage of the situation, gaining control of your application, system, or network, and can do any of the following:

- Read, add, delete, or modify your data or operating system,

- introduce a virus program that uses your computers and software applications to copy viruses throughout your network,

- introduce a sniffer program to analyze your network and gain information that can eventually be used to crash or corrupt your systems and network,

- abnormally terminate your data applications or operating systems,

- disable other security controls to enable future attacks. 
- The best way to prevent MIM and application layer attacks is to use a secure way.

\subsubsection{TCP replay attack}

A replay attack is when a hacker uses a sniffer to grab packets off the wire. After the packets are captured, the hacker can extract information from the packets such as authentication information and passwords. Once the information is extracted, the captured data can be placed back on the network or replayed. Some level of authentication of the source of event generator can help stop TCP replay attacks.

\subsubsection{Attacks on ONS}

ONS is a service that, given an EPC, can return a list of network-accessible service endpoints pertaining to the EPC in question. ONS does not contain actual data regarding the EPC; it contains only the network address of services that contain the actual data. This information should not be stored on the tag itself; the distributed servers in the Internet should supply the information. ONS and EPC help locate the available data regarding the particular object.

\subsubsection{Known threats to DNS/ONS}

Since ONS is a subset of Domain Name Server (DNS), all the threats to the DNS also apply to ONS. There are several distinct classes of threats to the DNS, most of which are DNS-related instances of general problems; however, some are specific to peculiarities of the DNS protocol.

- Packet Interception-Manipulating Internet Protocol (IP) packets carrying DNS information Includes MIM attacks and eavesdropping on request, combined with spoofed responses that modify the "real" response back to the resolver. In any of these scenarios, the attacker can tell either party (usually the resolver) whatever it wants them to believe.

- Query Prediction-Manipulating the Query/Answer Schemes of the User Datagram Protocol $(U D P) / I P$ Protocol These ID guessing attacks are mostly successful when the victim is in a known state.

- Name Chaining or Cache Poisoning Injecting manipulated information into DNS caches.

- Betrayal by Trusted Server Attackers controlling DNS servers in use.

- Denial of Service (DOS) DNS is vulnerable to DOS attacks. DNS servers are also at risk of being used as a DOS amplifier to attack third parties.

- Authenticated Denial of Domain Names

\subsubsection{ONS and confidentiality}

There may be cases where the Electronic Product Code (EPC) of an RFID tag is regarded as highly sensitive information. Even if the connections to EPCIS servers were secured using Secure Sockets Layer (SSL)/Transport Layer Security (TLS), the initial ONS look-up process 
was not authenticated or encrypted in the first place. The DNS-encoded main part of the EPC, which identifies the asset categories, will traverse every network between the middleware and a possible local DNS server in clear text and is susceptible to network taps placed by internet service providers (ISPs) and governmental organizations.

\subsubsection{ONS and integrity}

Integrity refers to the correctness and completeness of the returned information. An attacker controlling intermediate DNS servers or launching a successful MIM attack on the communication could forge the returned list of Uniform Resource Identifiers (URIs). If no sufficient authentication measures for the EPCIS are in place, the attacker could deliver forged information about this or related EPCs from a similar domain.

\subsubsection{ONS and authorization}

Authorization refers to protecting computer resources by only allowing the resources to be used by those that have been granted the authority. Without authorization, a remote attacker can do a brute-force attack to query the corresponding EPCIS servers until a match is found. In case the complete serial number is not known, the class identifier of the EPC may be enough to determine the kind of object it belongs to. If using the EPCglobal network becomes ubiquitous and widespread, the attacker could add fake serial numbers to the captured, incomplete EPC and query the corresponding EPCIS servers to find a match. This can be used to identify assets of an entity, be it an individual, a household, a company, or any other organization. If you wore a rare item or a rare combination of items, tracking you could be accomplished just by using the object classes.

\subsubsection{ONS and authentication}

Authentication refers to identifying the remote user and ensuring that he or she is who they say they are.

\subsubsection{Mitigation attempts}

- Limit Usage Use the ONS only in intranet and disallowing any external queries.

- VPN or SSL Tunneling With data traveling between the remote sites, it needs to be exchanged over an encrypted channel like VPN or SSL Tunneling.

- DNS Security Extensions (DNSSEC) ensure the authenticity and integrity of DNS. This can be done using Transaction Signatures (TSIG) or asymmetric cryptography with Rivest, Shamir, \& Adleman (RSA) and digital signature algorithms (DSAs).

\subsection{Risk and vulnerability assessment}

The assessment of risks and vulnerabilities go hand in hand. To begin evaluating your system, you need to ask questions regarding the assessment and tolerance of the risks: what types of information are you talking about at any given point in the system and what form is 
it in? How much of that information can potentially be lost? Once these risks are evaluated, you can begin to plan how to secure it. A good way to evaluate the risk is to ask five classic investigative questions: "who?", "what?", "when?", "where?" and "how?"

- Who is going to conduct the attack or benefit from it? Will it be a competitor or an unknown group of criminals?

- What do they hope to gain from the attack? Are they trying to steal a competitor's trade secret? If it is a criminal enterprise, are they seeking customers' credit card numbers?

- When will the attack happen? When a business is open 24 hours a day, 7 days a week, it is easy to forget that attacks can occur when you are not there.

- Where will it take place? Will the attack occur at your company's headquarters or at an outlying satellite operation? Is the communications link provided by a third party vulnerable?

- How will they attack? If they attack the readers via an RF vulnerability, you need to limit how far the RF waves travel from the reader. If the attacker is going after a known vulnerability in the encryption used in the tag reader communications, you have to change the encryption type, and, therefore, also change all of the tags.

\subsubsection{Type of RFID risks}

RFID technology enables an organization to significantly change its business processes to:

- Increase its efficiency, which results in lower costs.

- Increase its effectiveness, which improves mission performance and makes the implementing organization more resilient and better able to assign accountability, and

- Respond to customer requirements to use RFID technology to support supply chains and other applications.[16]

This section reviews the major high-level business risks associated with RFID systems so that organizations planning or operating these systems can better identify, characterize, and manage the risk in their environments. The risks are as follows:

Business process risk - direct attacks on RFID system components potentially could undermine the business processes the RFID system was designed to enable. For example, a warehouse that relies on RFID to automatically track items removed from its inventory may not be able to detect theft if the RFID system fails.

Business intelligence risk - an adversary or competitor potentially could gain unauthorized access to RFID-generated information and use it to harm the interests of the organization implementing the RFID system. For example, an adversary might use an interrogator to determine whether a shipping container holds expensive electronic equipment, and then target the container for theft when it gets a positive reading.

Privacy risk - the misuse of RFID technology could violate personal privacy when the RFID application calls for personally identifiable information to be on the tag or associated with 
the tag. For example, if a person carries products that contain RFID tags, those tags may be surreptitiously read by an adversary. This could reveal that person's personal preferences such as where they shop, or what brands they buy, or it might allow them to track that person's location at various points in time.[16]

Externality risk - RFID technology potentially could represent a threat to non-RFID networked or collocated systems, assets, and people. For example, an adversary could gain unauthorized access to computers on an enterprise network through Internet Protocol (IP) enabled interrogators if the interrogators are not designed and configured properly. Multiple RFID interrogators operating in a confined space may cause hazards of electromagnetic radiation to fuel, ordinance or people in the vicinity.

\subsubsection{Risks in supply chain management and tracking applications}

Tracking applications are used to identify the location of an item, or more accurately, the location of the last interrogator that detected the presence of the tag associated with the item. An example of an intentional attack on an RFID business process is cloning, which occurs when an adversary reads information from a legitimate RFID tag and then programs another tag or device to emulate the behavior of the legitimate tag. Another attack on an RFID business process would be removing a tag from the item it is intended to identify and attaching it to another unrelated item.

Supply chain management involves the monitoring and control of products from manufacture to distribution to retail sale. Supply chain management typically bundles several application types, including asset management, tracking, process control, and payment systems. Supply chain systems record information about products at every stage in the supply chain. Ideally, tags are affixed to products during the manufacturing process or soon afterward. As a product moves through the supply chain, to the customer, and to post-sale service, the tag's identifier can be used by all supply chain participants to refer to a specific item.

In addition, supply chain systems that use active tags can track larger objects such as cargo containers. Tags on these containers can store a manifest of the items shipped in each container. This manifest can be automatically updated when items are removed from the container. Potential problems are not just limited to the RF subsystem. If the network supporting the RFID system is down, then the RFID system is likely down as well. In supply chain applications, network failures at any point in the chain have the potential to impact the business processes of any subsequent link in the chain. For example, if a supplier is unable to write manifest data to a tag, then the recipient cannot use that data in its operations even if its RFID interrogators and network infrastructure are fully functional. Servers hosting RFID middleware, databases, analytic systems, and authentication services are all points of failure.

Any efforts to assess business process risk need to be comprehensive, because such a wide variety of potential threats exist. All of these threats have the potential to undermine the supported business process and therefore the mission of the implementing organization.[3] 


\section{RFID in procedural conditions of logistic operators}

Supply chain can be defined as the parts that are involved, directly or indirectly, in fulfilling a customer request (Chopra and Peter 2007). By this definition, it can be seen that a supply chain consists of manufacturers, warehouses, retailers, transporters, and customers. The purpose of a supply chain is to maximize the value generated for the customer; namely, maximizing the difference between the final product worth and the total expended by the supply chain to provide the product to the customer.

In order to succeed, the supply chain must be conducted to minimize the costs incurred. Supply chain management (SCM) is responsible for optimizing the flows within its operational stages which include raw materials, manufacturing, distribution, and transportation in order to minimize the total cost of the supply chain. SCM is a unification of a series of concepts about integrated business planning that can be joined together by the advances in information technology (IT) (Shapiro 2007), yet many companies have not completely taken advantage of this process.

In today's world, the competition between companies, more demanding customers, and reduced margins make the scenario more difficult for companies to succeed, to this context, SCM is an important practice for companies that want not only to keep in business but also have their results optimized and meet the clients' expectations.

Responsiveness in the supply chain has gained importance and it is a trend that apparently will dictate future decisions regarding supply chain design. According to Kovack, Langley, and Rinehart (1995), the themes that will have influence on logistics on the near future are:

- Strong corporate leadership will enhance logistics value through focusing on efficiency, effectiveness, and differentiation.

- Value realization requires marketing of logistics capabilities within the company and to external customers.

- Emphasis on the "scientific" aspect of logistics management in order to enhance the "art" of creating customer satisfaction. Enhancing logistics value through integrating product, information, and cash flows for decision-making linking external and internal processes. Logistics value enhanced by ownership of responsibility internally and externally to the firm.

- Focus of successful companies is to create internal value for their organizations and external value for their suppliers and customers.

From these themes, it can be seen that SCM plays and will continue to play an active role in successful companies' routines. In order to achieve better results in the supply chain and better responsiveness to customers' necessities, new techniques such as real-time inventory and dynamic supply chain need to be developed. 


\subsection{Transportation in logistics and SCM}

As a supply chain driver, transportation has a large impact on customer responsiveness and operational efficiency. Faster transportation allows a supply chain to be more responsive but reduces its efficiency. The type of transportation a company uses also affects the inventory and facility locations in the supply chain. The role of transportation in a company's competitive strategy is determined by the target customers. Customers who demand a high level of responsiveness, and are willing to pay for the responsiveness, allow a company to use transportation responsively. Conversely, if the customer base is price sensitive, then the company can use transportation to lower the cost of the product at the expense of responsiveness. Because a company may use transportation to increase responsiveness or efficiency, the optimal decision for the company means finding the right balance between the two.

The transportation design is the collection of transportation modes, locations, and routes for shipping. Decisions are made on whether transportation will go from a supply source directly to the customer or through intermediate consolidation points. Design decisions also include whether multiple supply or demand points will be included in a single run or not. Also, companies must decide on the set of transportation modes that will be used.

The mode of transportation describes how product is moved from one location in the supply chain network to another. Companies can choose between air, truck, rail, sea, and pipeline as modes of transport for products. Each mode has different characteristics with respect to the speed, size of shipments (parcels, cases, pallet, full trucks, railcar, and containers), cost of shipping, and flexibility that lead companies to choose one particular mode over the others. Typical measurement for transportation operations includes the following metrics:

- Average inbound transportation cost, or the cost of bringing product into a facility as a percentage of sales or cost of goods sold (COGS). Cost can be measured per unit brought in but is typically included in COGS. It is useful to separate this cost by supplier.

- Average incoming shipment size measures the average number of units or dollars in each incoming shipment at a facility.

- Average inbound transportation cost per shipment measures the average transportation cost of each incoming delivery. Along with the incoming shipment size, the metric identifies opportunities for greater economies of scale in inbound transportation.

- Average outbound transportation cost measures the cost of sending product out of a facility to the customer. Cost should be measured per unit shipped, oftentimes measured as a percentage of sales. It is useful to separate this metric by customer.

- Average outbound shipment size measures the average number of units or dollars on each outbound shipment at a facility.

- Average outbound transportation cost per shipment measures the average transportation cost of each outgoing delivery. 
- Fraction transported by mode measures the fraction of transportation (in units or dollars) using each mode of transportation. This metric can be used to estimate whether certain modes are overused or underutilized.

- The fundamental trade-off for transportation is between the cost of transporting a given product (efficiency) and the speed with which that product is transported (responsiveness). Using fast modes of transport raises responsiveness and transportation cost but lowers the inventory holding cost.

\subsection{Information technology and SCM}

It is no surprise that IT played a big role in enabling many processes and ideas in Supply Chain Management (SCM) that seemed impossible in earlier years. The first advance was the decreasing of inventory levels by managers abandoning rules of thumb and adopting the setting of inventories based on service level desired and historical demand (Shapiro 2007). IT allowed the analysis of a great quantity of units and the process of recalculating the inventory level as the demand changed. This ability to analyze inventory needs made the companies more agile while decreasing inventory levels and increasing service levels.

Another important fact that gave a great contribution to SCM was the electronic interchange (EDI). This technology allows the direct data interchange between companies using computers. EDI changed the relationship between the company and customers, with its suppliers, and also with the employees. The ability of trading data almost instantly across the supply chain gave companies the ability to manipulate more up-to-date information in a shorter period of time. This reduced the need for printing and transporting papers, enabled just-in-time practices, and helped to restructure logistics supply chain relationships. Together with EDI we can also mention the importance of the Internet in global business (Johnson et al. 1999).

Artificial intelligence systems are responsible for many advances achieved by society and by SCM as well. Computers can be programmed to execute routine functions and according to the rules imposed to the computer it can be capable of behaving an intelligent system that can execute complex activities in reduced time. This brought to logistics a much larger capacity of processing information and executing tasks. Many activities can operate without human interference and this converges to a more responsive and accurate supply chain (Johnson et al. 1999).

Some technologies, discussed later in this chapter, can be used to make real-time adjustments to the supply chain. Those adjustments could be due to many events such at manpower shortages or equipment breakdowns. For example, if a problem occurs with a truck or the road conditions change due to weather, the system, supplied with this updated information, should be able to make the necessary corrections to the transportation routes of other trucks to compensate for the truck failure.

This system would be very useful for natural disasters such as Hurricane Katrina. With realtime information, the system would reallocate transportation and production. This kind of modeling would reduce the response time for such events from months or weeks to days or 
even hours. This system can also be expanded to urban transportation within a city or long distances between two cities.

\subsubsection{Real-time technologies}

Radio frequency identification (RFID) and global positioning systems (GPS) are emerging technologies that will allow for real-time data collection to assist with decision support in SCM. RFID has a wide variety of applications. Some examples of RFID uses are library checkout stations, automatic car toll tags, animal identification tags, and inventory systems. Real-time data collected using RFID allows a supply chain to synchronize reorder points and other data. Real-time information can also be used to design and operate logistical systems on a real-time basis. GPS is currently used solely as a means to locate equipment and derive navigation directions.

An RFID system consists of a reader, tags, and an air interface. The reader, also known as an interrogator, sends out a signal through an antenna. This signal is usually in the form of an electromagnetic wave, so a direct line of sight is not needed to read the information on the tag. This is a major advantage of RFID. The signal is received by the tag and a response signal is sent back to the reader. This response signal contains a unique identifier associated with a tag. The response signal can be powered in two ways corresponding to the type of tag. Passive tags utilize the energy of the original signal to send a response signal back to the reader. Passive tags have a limited amount of energy to power the response signal. Therefore, the amount of information transmitted by a passive tag is fairly small, quite similar to the information carried in a bar code. Active and semi-active tags use energy from an attached battery to power the response signal. The use of the embedded battery allows the response signal to contain more information and travel farther. The reader receives the response signal, decodes it, and sends that information to a database. Often the information in the response signal is connected to additional information in the database.

RFID technology can be used throughout the supply chain in order to promote visibility. This visibility helps coordinate actions between entities in the supply chain. Figure 4 shows the relationships within the supply chain that can be affected by the implementation of the RFID technologies. An example of RFID implementation is the use of active tags for detecting tampering and monitoring security of maritime containers. Those types of tags also have the tracking advantages of RFID and can be used to improve operations management. Those tags can be seen in Figure 5.

GPS systems consist of a series of receivers and satellites that orbit the Earth-GPS works by calculating the distances from a receiver to a number of satellites. With each distance between a receiver and satellite, the number of possible locations is narrowed down until there is only one possible location. A receiver must calculate its distance from at least three satellites to determine a location on the surface of the Earth. However, four satellites are usually used to increase the location accuracy (Dommety and Jain 1996). This process of location would be controlled by the positioning module of GPS system. An average GPS positioning and navigation system would also have the following modules: 


\section{- Digital map database,}

- Map matching,

- Route planning and guidance,

- Human-machine interface,

\section{- Wirelesscommunication.}

\section{RFID Transportation Systems Applications}

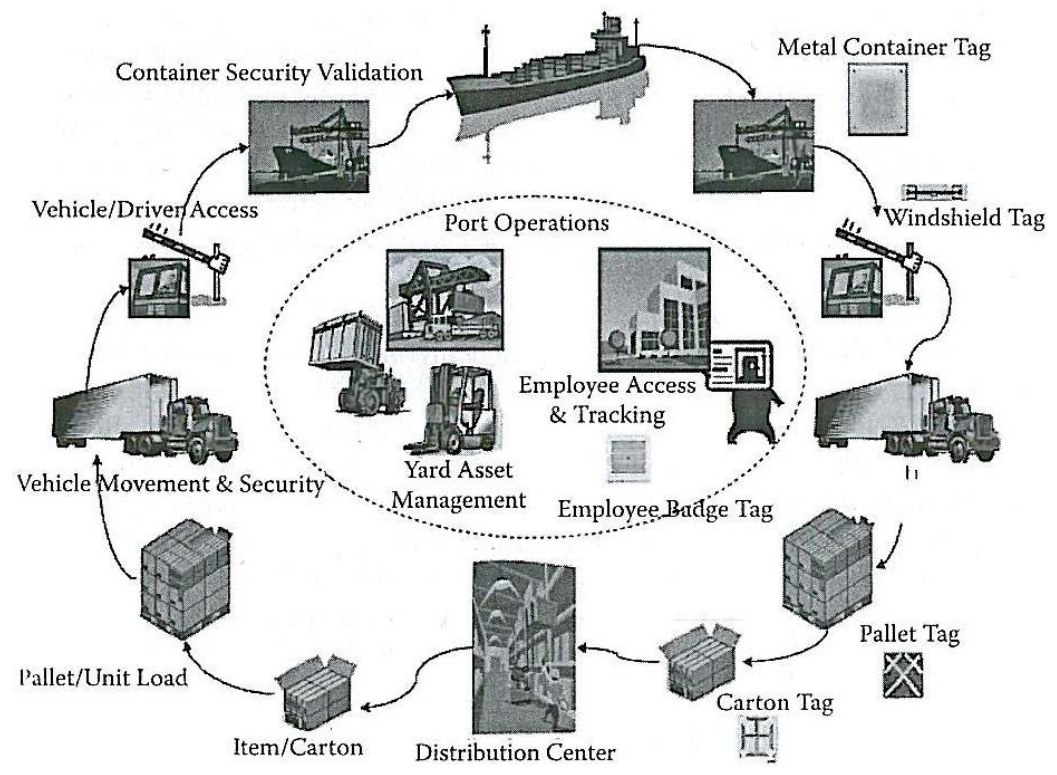

Figure 4. Integrated supply chain with RFID (Source: SAVI Technology)

There are three positioning technologies that can be used: radio wave-based positioning, dead-reckoning, and signpost. The use of GPS for navigation can have direct and indirect impacts on intelligent transportation systems. GPS navigation systems can provide information about local surroundings. Also, emergency personnel can be provided with a precise location for situations, thus reducing response times. Asset tracking is one of the most popular uses of GPS. One of the limitations of GPS is that receivers cannot communicate with satellites when indoors (Feng and Law, 2002).

RFID and GPS are radio wave-based technologies that are currently used by many organizations. RFID is primarily used in inventory and material handling processes. Tags are placed on items. When these items pass by checkpoints where readers are located, the tag is read and the appropriate action can be taken. Real-time inventory can be kept by moni- 
toring tag reads at strategic points like loading docks. RFID can also be useful in material handling. Items on a conveyor can be diverted at the appropriate times based on the information received from the RFID tag. GPS is primarily use to track assets such as vehicles and other expensive equipment. For example, if a truck breaks down, it is possible to locate the truck and get the shipment moving again in a fraction of the time it would take with a GPS receiver.

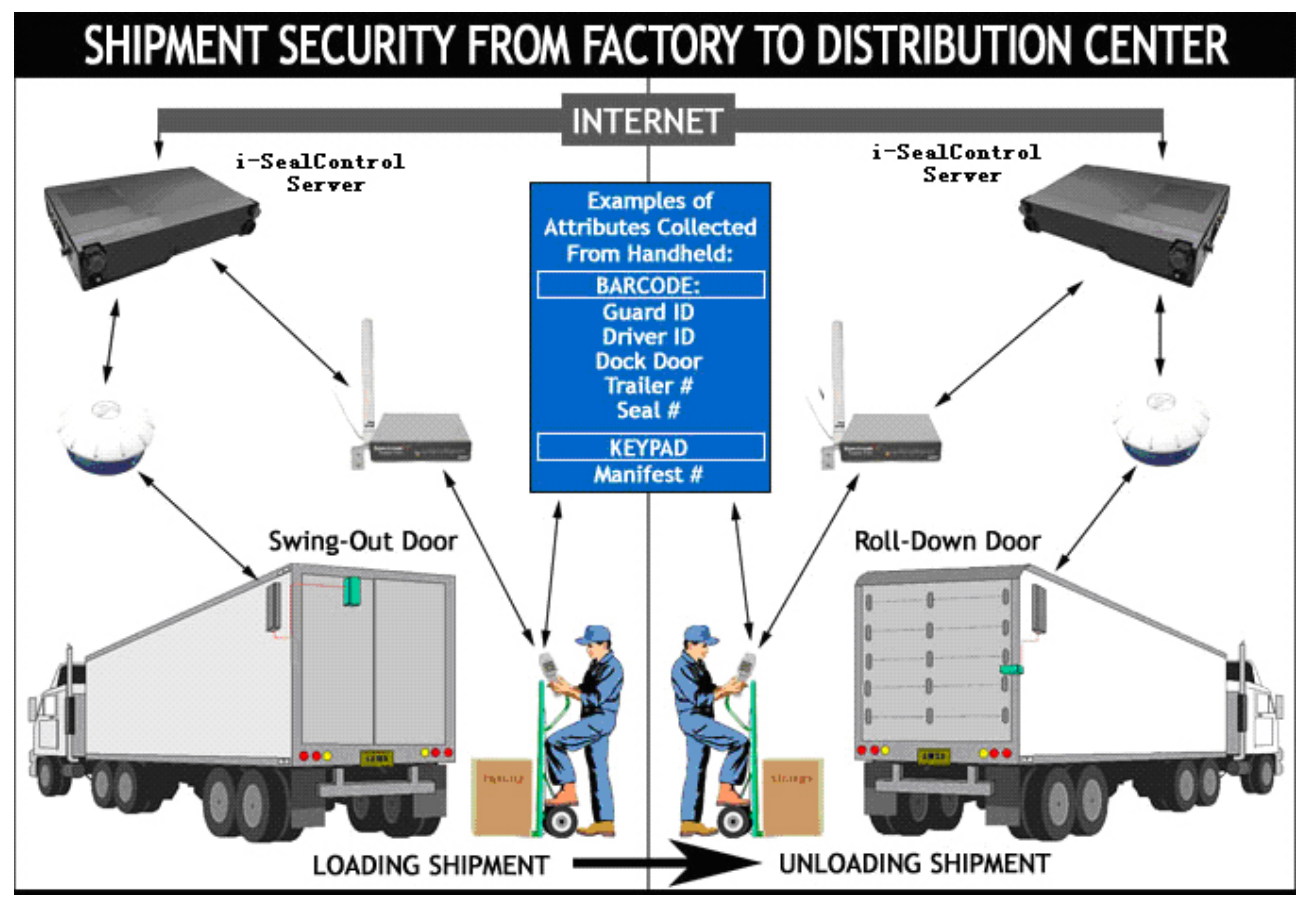

Figure 5. RFID Container Seal (Source : SAVI Technology)

\section{Summary of RFID and Information Enablers}

This section provides understanding of key technologies and how all the technologies differ and how they can be integrated to work for operational effectiveness. This will allow warehouse management system algorithms such as "bucket brigade" calculations, picking route optimization, and other effective system updates that will improve operations. Further insights into safety stock minimization, customer order optimization, and pick/stock labor minimization will be affected and discussed later.

\subsubsection{RFID Provides timely visibility in logistics}

RFID supports information in the supply chain by enabling visibility. The concept of visibility describes the ability of anyone, including customers, to have access to inventory, orders, 
raw materials, and delivery points at any time. Visibility is currently [provided by a mixture of automatic identification, or auto-ID, technologies such as bar codes, smart labels, ISBN, and UPC codes, along with others. The opportunity for RFID is that its non-line-of-sight scanning, the integration of the aforementioned auto-ID identifiers into RFID nomenclature, and the push for standardized technology protocols will provide large supply chain savings. The real-time nature of RFID is considered a benefit and currently a challenge. The benefit is that you have the latest information to make the best decisions; the drawback is that the amount of data currently presents a data storage problem for operational systems.

Better visibility provides reduced inventory, labor and assets management using inventory policies, scheduling, and decision support system information. This is exemplified by the fact that:

- RFID supports reduced inventory costs with more effective labor policies

- RFID supports labor reduction with more effective scheduling

- RFID supports the reduction of expensive assets such as facilities, trucks, containers, and railroad time because of more accurate information in decision support systems. The ability for RFID to provide timely information and visibility into the supply chain are based on three components of RFID technologies. They are

- Automatic data capture,

- Real-time information

\section{- Real-time location system.}

The RFID enabling technologies diagram shown in Figure 6 represents these components as interconnecting orbits.

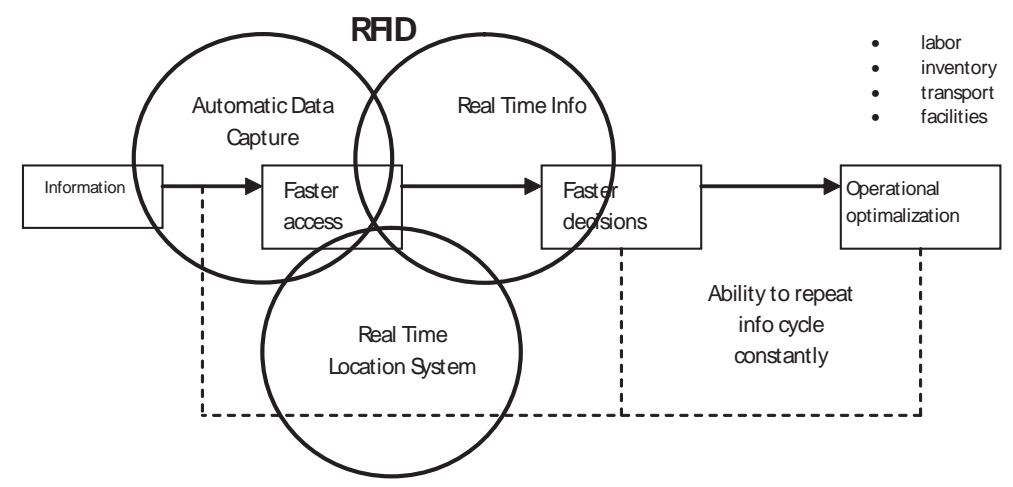

Figure 6. RFID-enabling technology

The figure also shows how RFID supports timely information in the supply chain by enabling information to be accessed faster. This implies that faster decisions can be made, which 
produces operational optimization that can be effectively repeated. In the figure, one of the boxes represents the RFID information flow. The ability to allow resident information collected automatically in real-time leads to faster, more effective decisions is where RFID shows future promise. Business costs are reduced as operations become more productive by reducing labor, transportation, and facility cost of moving inventory in the supply chain and postal services.

Many organizations see that the benefit of using RFID is that they can effectively manipulate inventory. Inventory exists in the supply chain because of the variance between supply and demand. This variance is necessary for manufacturers where it is economical to manufacture in large lot quantities and then store for future sales. The variance is also present in retail stores where inventory is held for future customer demand. Oftentimes businesses suggest that inventory is a marketing vehicle creating demand by passing customers. The main role inventory plays is to satisfy customer demand by having product available when the customers want it. Another significant role that inventory plays is reducing cost by exploiting economies of scale that may exist during production and distribution. Given that economy of scale is believed to have such a large impact on inventory, we will present some relevant information regarding inventory in the supply chain.

RFID is essentially in the same position occupied by mobility and wireless technology a few years ago. It is poised to spark a global revolution-in supply chain visibility and management. Using RFID in pivotal points in the supply chain can help enable a vision of having goods available to customers at the right place and at the right time. RFID technology is an enabler of this vision aiding the synchronization between physical and information flow of goods across the supply chain from Manufacturer to Retail Outlet, represented on figure 7 . [1]

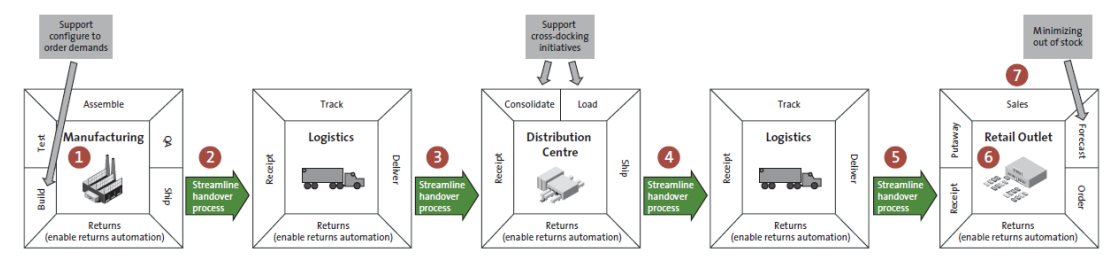

Figure 7. Supply chain containing RFID technology

\section{Manufacturing}

As goods travel down the production line, RFID tags are physically applied and a unique ID is written and then validated for quality assurance purposes. The unique ID is automatically associated to the product/order details to facilitate further tracking and exception management.

During the pallet build process; goods (e.g cases) are automatically identified to aid with customer order configurations. Finally, pallets are identified and tracked as they are delivered to the staging area ready for shipment. 


\section{Manufacturer - Logistics pickup}

As the logistics vehicle arrives at the loading dock, the RFID reader positioned at the loading dock communicates with the unique RFID tag to confirm that the logistics vehicle is authorised to pickup goods. Upon approval, pallets leaving the loading dock communicate with the RFID reader to alert B2B systems (ASN) and ERP systems to initiate electronic transactions, proof of pickup and potentially shipment invoicing.

\section{Logistics delivery - Distribution centre (dc)}

As the logistics vehicle arrives at the Distribution Centre, the RFID reader and middleware initiates an event that captures the unique ID from the RFID tag, triggering the arrival of the manifest to initiate automatic routing of the goods to the next logistics vehicle (load consolidation).

\section{Distribution centre - Logistics delivery}

As pallets are loaded onto the logistics vehicle the RFID reader positioned above the loading dock communicates with the RFID tags. The RFID tags broadcast their unique ID to the reader and via the RFID middleware transfer information to ERP systems indicating that the manifest is loaded.

\section{Logistics delivery - Retail outlet}

As the shipments of goods arrive at the receiving dock (again being detected by RFID readers), Retail ERP systems are updated to manage inventory levels (automatically, accurately and at low cost) and initiate B2B messages to Suppliers to commence invoicing.

\section{Retail outlet - Customer}

As items are removed at shelf level, the RFID reader can automatically detect the event and via the RFID middleware, initiate additional product supply requests. With such a system in place, the need to maintain costly volumes in remote warehouses is almost eliminated. At this point of the process, the customer is initiating direct demand generation on the supply chain management process.

\section{Customer}

Rather than wait in line for a cashier, the customer simply walks out the door with the purchase. A reader built into the door recognises the items in the cart by unique ID's. A swipe of the debit or credit card and the customer is on their way.

\subsubsection{Future technologies}

Current applications of RFID and GPS systems have allowed for more effective tracking of inventory and assets. These technologies can be used in conjunction, but the data has to be captured and written to a database to be correlated to other tags or receivers. If these technologies can be combined to produce hybrid systems, greater gains can be achieved. One focus of research is the nesting of GPS receivers and various RFID tag types. If tags and receivers were able to communicate with one another, even more accurate real-time data 
collection could be achieved during transportation. This would also reduce equipment costs because fewer readers would be required. The nesting would follow the form in Figure 8.

If these technologies can be nested, it will allow the information in a bar code or a passive RFID tag to be collected by an active tag. This information could then be combined with the information contained within the active tag and transferred to a GPS receiver. The GPS receiver could then send not only its location but all of the information about the cargo being shipped (Reade and Lindsay 2003). A possible application of this nested technology approach would be in the railroad industry. Currently, there are two passive RFID tags attached to the sides of all railcars in the United States. In addition, most railroads use GPS receivers to track locomotives. If nesting became possible, implementation would be easy. Active tags could be used to capture the information correlated to the cargo in all of the railcars and transmit it to the GPS receiver and thus to the inventory databases.

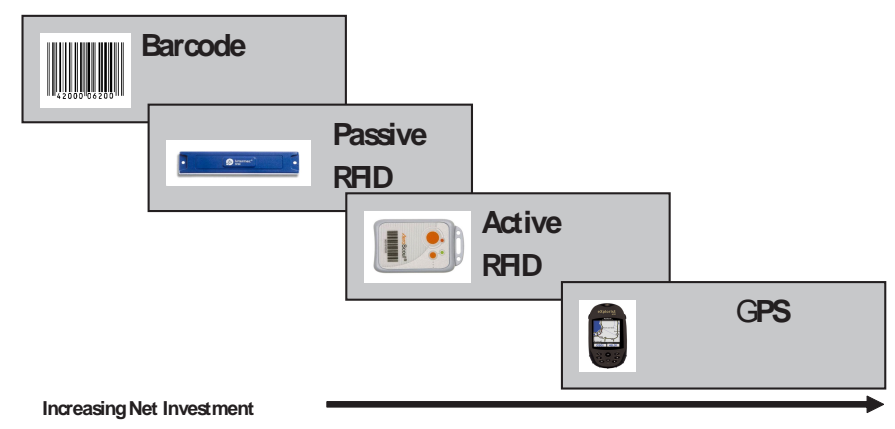

Figure 8. Nesting diagram

In addition to nesting technologies, more advanced tags can be developed to allow more detailed data collection. Tags that utilize sensors to capture and write data to the tag are being developed. Some tags have been developed but are still very unreliable. These sensor tags could be used to monitor physical parameters, like temperature and humidity, as well as security parameters. The main problem faced by these passive sensor tags is the limited power supply. The sensor cannot use any energy while outside the range of the reader. Also, the amount of energy available while in read range is very small. This limits possible measurement techniques (Want 2004). With these sensor tags, perishable goods could be monitored to guard against possible safety issues. This could include salmonella outbreaks caused by frozen chicken reaching too-high temperatures for too long and medications being held at temperatures that reduce potency.

\section{AIDC and mobile technologies in postal sector}

This part primarily deals with identification of postal items and transport units in logistic chain of postal operators. Nowadays, the identification is carried through barcodes and opti- 
cal character recognition. In this article we would like to specify, how can be transport units identified in the transmission process by RFID technology. In the carriage of postal items is necessary to decide what type of transport is used for that purpose, what the flows of items are and what their intensity is.

The part described scheme of the transport process, including planned technology and there is also simulated a real postal process in conditions close to operational.

An unavoidable part of today is a dynamic development in the field of mobile technologies, their everyday use and application of the processes, which largely supports the level of quality of postal services and thereby strengthening the market positions of individual postal operators. This area is even more pertinent that in all countries of the European Union since 1 January 2013 approved the postal market and postal services. In this respect, it is necessary to include postal processes embarked on new technologies to ensure the competitiveness of the national postal operator and alternative providers.

RFID technology has been selected by an international post corporation (IPC) to test deliverability (transit time) of items in 55 countries of the world (Slovak republic including). The requirement of transit time is defined by Universal Postal Services and applicable also for Slovak Post. Despite the RFID technology is being known and being improved for a long time, it is essential to define the standards and security requirements.

Besides efficiency, consolidation and globalization within the European Union, interoperability is one of key elements. It is the ability of information and communication systems (including the supported processes) to exchange data, share information and knowledge, which leads to standardization.

\subsection{Methods and aims}

For understanding of issue is should be analyze terms used. The availability of RFID components, GPS devices and possibility of using satellite navigation there is possible to create a relative effective infrastructure for improving management of transport process by post.

There is true, that personal correspondence is on the wane, the main reason is development of information technology especially Internet, but large part of using a postal services have a companies and therefore the services will remain an indispensable part of society.

\subsection{Structure}

When we focus on these connections, external influences on postal sector and potential current technologies there is important to analyze possibilities of automation individual processes, improve a transportation operating activities and ensure continuity in fulfilling the goals. These aims lead to satisfying of customers in area of provide post services at phase in the delivery of mail.

The aim of this part is refer on possible improve in this area. The most important term of category, which will use in individual chapters are: mobile technology, definition of means transport. 


\subsubsection{Mobile technology}

The classification of wireless technologies based on the distance or reach of the broadcast signal provides insight on their potential use. A condition of transport a date in broadcast systems and networks is communication without physical contact.One of the possible division of this system is on range of coverage:

- Global system - These systems coverage of territorial area. There we can speak on worldwide operating systems, which aren't dependent on a concrete application and their communication is carried through different protocol. (for example: Satellite communication systems, GPS)

- Metropolitan systems - These systems operate on lower geographic area. They usually operate at state level. (For example: The system based on wireless technology, Wi-Fi)

- Local systems - These systems operate at a distance, which include a several $\mathrm{cm}$ up to several hundred meters (For example: Bluetooth, RFID)

\subsection{Postal transport network}

The postal transport is most important part of process from submission of mail after its delivery to addresses with consistent set of quality standards for different types of mail. These standards are also based on the postal license and the requirement for quality by the universal postal services.

The postal transport network includes postal courses and infrastructure. While constructions of postal transport network are use a different systems and tools. The postal transport network is divided into three basic levels:

- district transport network (OPS) - this network connecting establishment with other facility of processing center area.

- regional transport network this(RPS) - this network connecting the main processing centers with district processing centers of own district.

- main transport network (HPS) - this network connecting the main processing centers, the main processing center with the district processing centers from different district of HSS. This network includes transport conclusion in international relations.

In the carriage of postal items is necessary to decide what type of transport used for that purpose, what are the flows of items and what is their intensity. Way to connect and type of vehicle depends on the following factors:

- density and organization of the postal network,

- flows of different types of postal items and their size,

- the carrying capacity of vehicles used,

- transport time of each species of postal items, 
- safety and effectiveness of postal traffic.

Processing of items is implemented in the workplace of the Slovak Post:

- HSS - main processing center - the facility providing treatment and quest items posting its area of perimeter, mail items addressed to your district and in transit in its dealings with OSS circuit, in contact with other HSS and OU,

- OSS - regional processing center - post office responsible for preparing and quest items posted at post offices in his own constituency and in transit in contact with your postal district and interacted with the HSS, the facility responsible for receiving, processing and quest items express postal services,

- selected post - post office responsible for preparing and quest items selected species within a specified range (usually as OSS),

- Exchange post - processing the shipment and ensure shipments to post offices exchange foreign postal administrations,

Regional hub as department of express service - establishment is responsible for receiving, processing and quest items express postal service.

\subsection{Characteristics of transport units and processes}

Characteristics of transport units - Slovak Post, a. s. used in the transport process shipments following shipping units: containers, leaf containers and postal bags. Containers are used in the transport process at HSS and OSS, on the local network using only postal letter case and postal bags.

Basic flow of transport processes are show on next figure including use a basic mobil technology in relevant stages.

The postal courses represent connection, which is set by transportation route with time data movement of vehicles used for carrying of postal mails. The postal courses are divided by the following criteria:

- The rail transport - used on carrying of postal mails through rail network. The conclusions are transport in wagon, which owned SP, a.s.

- The road transport - used road infrastructure for carrying conclusions. The conclusions are transport by vehicle, which own of SP, a. s.

- The fly transport - this type of transport is most advantageous for fast speed and overcoming large distance. The SP, a.s. used this type of transport on agreements with individual airlines. It only use for international postal mail transport.

The greatest part of transport postal mail is ensured by the road transport between main transport network (HPS), regional transport network (RPS) and district transport network (OPS). 


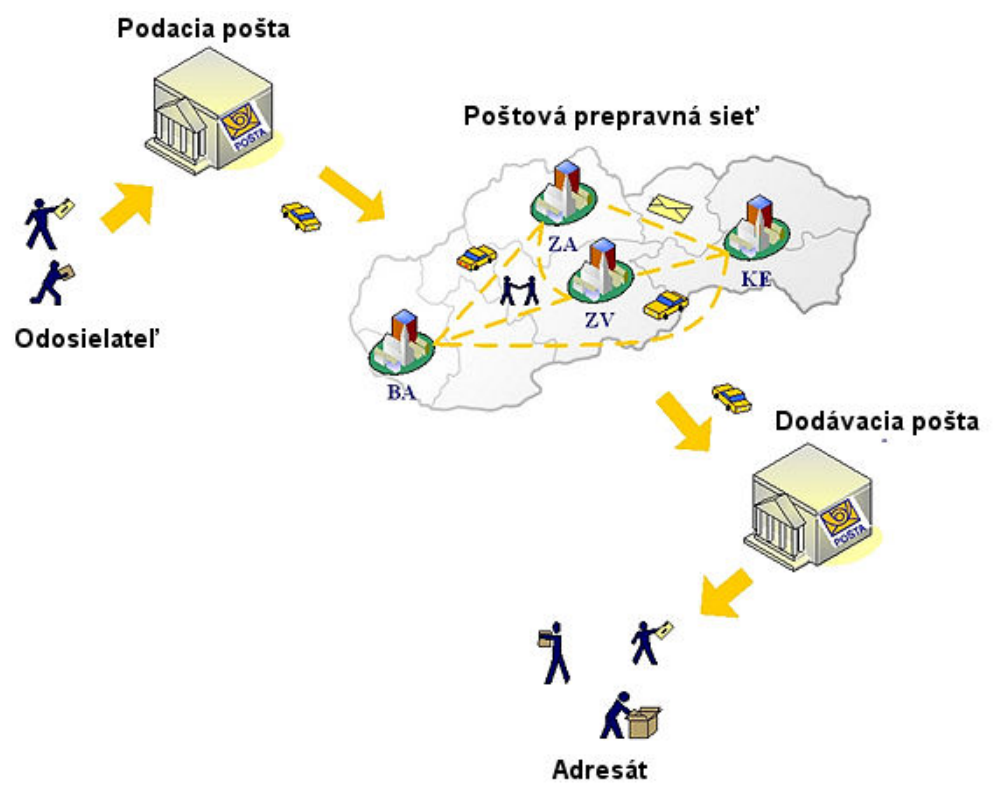

Figure 9. Simplified diagram of movement of the consignment of transmission network in Slovak

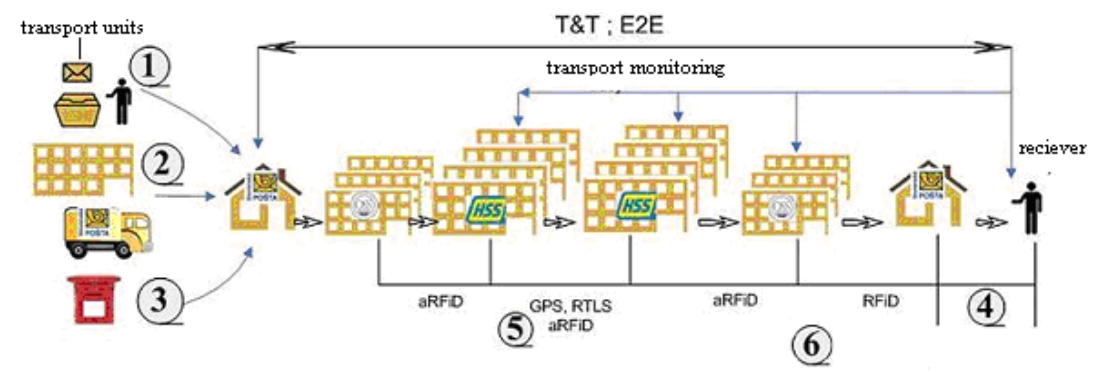

Figure 10. Scheme of the transport process, including planned technology

1. sender pass the post office at the counter

2. collecting expedition posting or accumulating courses,

3. pass through postal box,.

4. mobile technology - monitoring the transport process,

5. possibilities for optimizing routes for mail delivery

6. communication with the addressee. 


\subsubsection{Transport units}

The Slovak Post used the following transporting units in the transport process:

- container

- letter boxes

- bags

The postal operator has four types of containers for transport of letters and bags:

- platform truck - made by aluminum profiles connecting by PVC parts. This container is equipped by securing straps,

- stable structure track with rear wall and two side panels with wire grid 100x100mm,

- truck shipments on a very stable structure, floor frame and rugged steel profile galvanized steel thickness $1 \mathrm{~mm}$,

- folding platform truck made of steel profiles welded together by fasteners.

The containers are used in the transport processing at HSS and OSS. In the local postal network used only containers and bags.

\subsection{Design applications}

It is obvious that these systems are in a lot of cases combined and interrelated. In this design is emphasis on technology, which their using isn't common. There is mean GPS, Wi-Fi, GSM and more. On the figure, there are plans with this technology. Some of these technologies the postal operators used now and this is reason, why was this design focused on mail monitoring in transport processes on passive RFID technology.

For possible future use of the possibilities currently offered by some mobile technologies, we have tried outline Figure 11 scheme of the transport process, including the applicable technologies selected and purpose of their use:

aRFID such as active RFID technology - application within HSS and OSS use on monitoring containers a transporting units, optimization process and better evaluation quality of postal services,

- pRFID such as passive RFID technology - application between post office and sender/ addresses of postal mails. There is a lot of option of using,

- BC - bar code - barcode using by SP, a, s. at present,

- RTLS - monitoring mails, which are important or contain perishable content,

- GPS - route monitoring, possible specifying of delivery place for some type of mails,

- GSM - communication through mobile phone, information about mails, possible locate a place for delivery mails, possible pay for service through mobile phone 


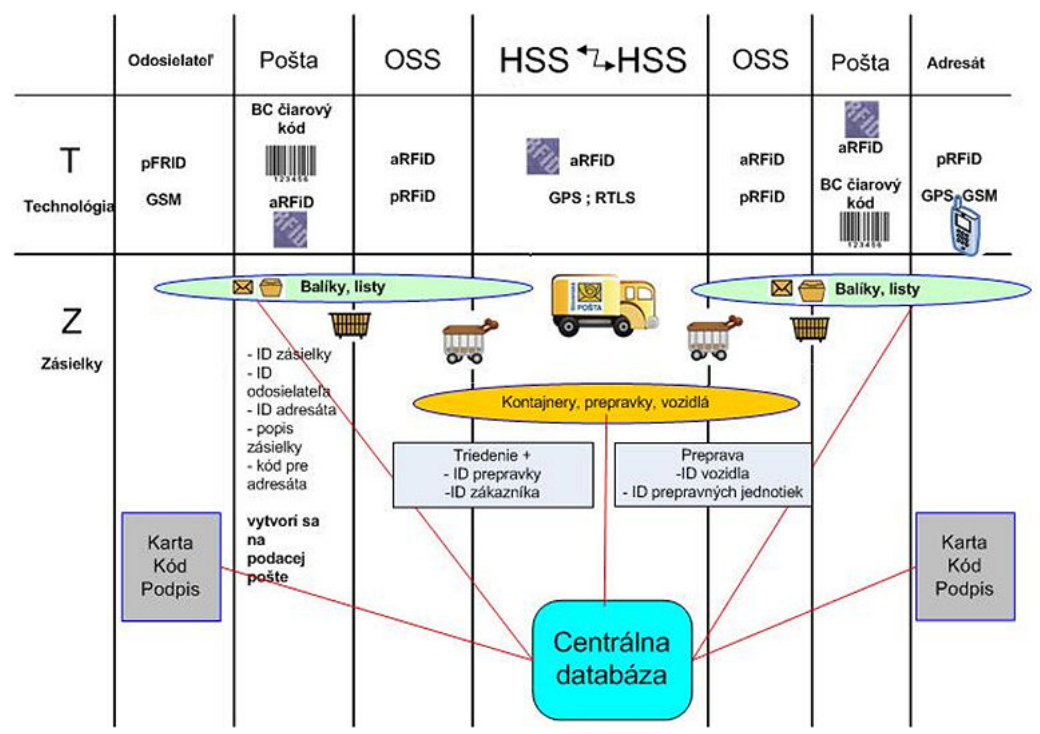

Figure 11. Scheme of the transport process, including the anticipated technologies

\subsection{Types of RFID application in conditions of postal processes}

The AMQM ${ }^{1 T M}$ Platform provides postal operators with a complete overview and effective traceability of mail volumes, parcels, mail bags, mail items, trucks, roll-containers and letter trays across the entire logistics chain. One key feature is automatic consignment system that associates the mail items to the containers carrying these items and to the trucks transporting these.

This solution can be based on various technologies such as: RFID, disposable RFID labels and bar codes, as well as combinations thereof. It also enable objective documentation of times of arrival and departure of vehicles, which postal containers are loaded/unloaded, vehicle load space management, real-time information on types of mail, quantities, times of arrival, delays or changes in transport times etc. With regard to postal operational systems, the following conditions must be taken into account:

- Rough industrial environments.

- Large volumes of goods and mail.

- Short time available for processing.

- High labor costs in connection with the daily operations.

- Large potentials in automation and streamlining of manual processes. 


\subsubsection{RFID-based vehicle management}

Tracking vehicles and trailers throughout the entire transport logistics chain provides considerable benefits to all parties involved, e.g. management, users and customers. The Vehicle and Trailer Tracking System is an advanced and effective IT system for monitoring and managing precise arrivals and departures of vehicles at specific points in the logistics chain.

The system is built on the experience and know-how acquired from supplying the world's largest and most widespread RFID network stretching across about 60 countries.

Implementing this system offers unique values. Examples of benefits:

- Fully automatic registration of vehicles - i.e. no manual work involved.

- Improved yard and vehicle management.

- Precise and objective record of exchange of goods between parties.

- Early warning on delays in transport to all parties.

- Precise feedback to transport planning systems.

- Improved vehicle maintenance routines.

- Cost savings in centers with real-time information available.

\subsubsection{Roll cage tracking and managing}

One of the main issues being addressed by the roll container tracking and managing project is need to take control of and better manage transportation assets. Another primary project requirement is to ensure that the required containers will be always available at the customers' premises and within postal operator facilities. This should overcome the tendency for planned or unplanned hoarding of roll containers that causes shortages elsewhere, especially at peak times.

Additionally, the lack of visibility of roll container whereabouts led to unnecessary loss since it was not possible to identify where the roll containers disappeared and hence forced expensive purchase of new roll containers to meet the customer service level agreements. System of the monitoring and managing roll cages includes tag (active or passive, it depends of application), that is placed on a side or on the bottom of the container (Figure 12), it also includes a handheld terminal solution for consignment of roll container, product and destination enabling load control on all roll containers (Figure 13).

The result is avoiding miss-sending and has real-time $t$ volume forecasting into all facilities in the network providing efficient and on time production and distribution. Also a handheld terminal solution designed for track and trace of all individual parcels is a part of the solution providing key customers with shipment visibility throughout the whole logistic network. Miss-shipments are prevented by load-control. 


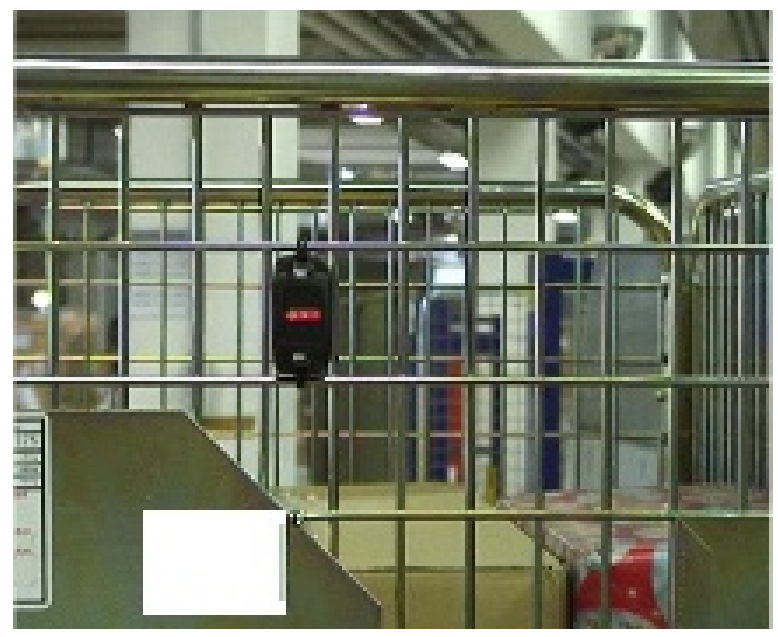

Figure 12. RFID tag placed on the container

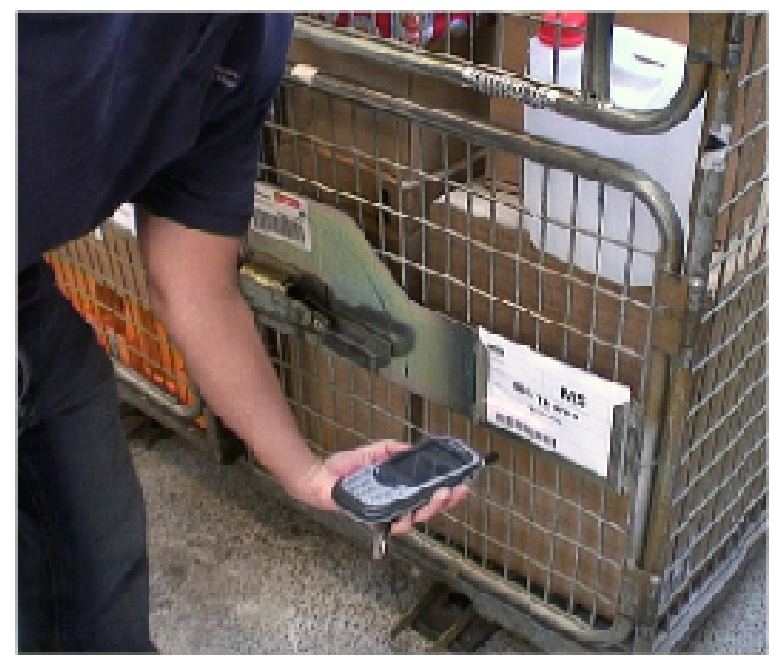

Figure 13. Handheld terminal

When a roll container is ready for dispatch, the roll container is scanned for destination and product type. If the roll container is lead through a gate not matching the destination, an alert will immediately help correct the mistake. Solution must include Asset Management software platform enabling full, real-time transparency of the location of each roll container and can be also used to track specific mail and parcel transports. [6]. Implementing this system offers unique values. Examples of benefits: 
- Improves availability and load balance throughout the logistics chain.

- Prevents hoarding of roll-containers.

- Minimizes losses.

- Helps to improve supply chain efficiency.

- Provides the ability to monitor the transported delivery time of goods.

- Helps to improve service and maintenance.

\subsubsection{Letter tray tracking}

Tracking and tracing letter trays throughout the entire postal logistics chain provides benefits to postal customers, employees and management. The trays are automatically registered in the postal logistics by means of RFID technology. Each letter tray has a tag that communicates and transmits information to the reader in Real-time load control (Figure 14). Now it is possible for the postal operators to reuse the same RFID network to track \& trace postal letter trays. This new opportunity is a fast pay-back investment with many unique advantages to postal operators worldwide.

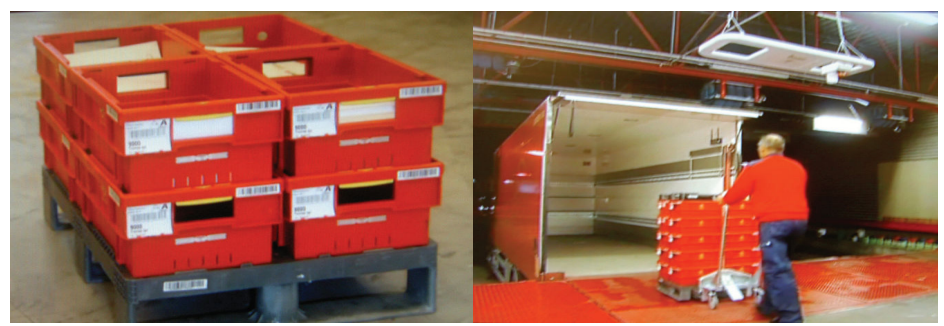

Figure 14. RFID tags on letter trays and Real-time load control

Key Benefits:

- Better utilization of postal letter trays.

- Possibility to analyze though-put times of mail and letter trays at distribution centre.

- Knowing the location of trays improves their availability throughout the entire logistics chain.

- Knowing the location and contents of trays improves the possibility of managing the tray sorting process right on time.

- On automatic handling systems, such as tray sorters, the reading rate can be improved dramatically compared to that of bar codes - reducing manual intervention.

- Being able to identify trays helps to improve service and maintenance. 


\subsubsection{Mail bag tracking}

Mail bags are widely used all over the world for transporting letters. The use of the mail bags differs between postal operators from transporting standard letters, to added value letters or to being used in closed customer loops. Each mail bag has a passive RFID tag that contains information about letters, which are inside the bag and some other additional information useful for sorting and other postal processes (Figure 15).

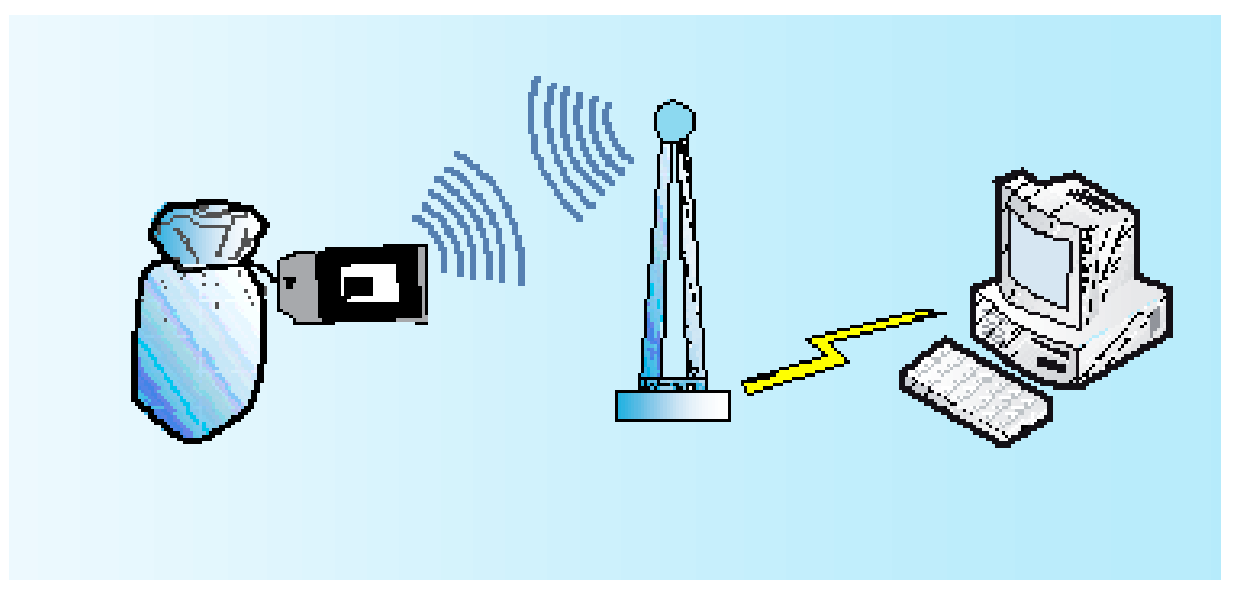

Figure 15. RFID tag placed on Mail Bag

Independent of how each postal operator is using the mail bags, tracking them can improve their competitiveness by means of:

- Optimization of processes.

- Optimization of routes.

- Control of Quality of Service.

- Internal documentation of handovers.

- Customer documentation.

Tracking solution is based on:

- Bar codes.

- Active RFID tags.

- Passive RFID tags.

- Hand-held terminals or PDAs.

- Automatic scanners. 


\section{The impact of the operational characteristics on the readability in postal sector}

In this part we show the reality of using RFID technology to identify the letter by specific analysis of the legibility of letters in the crate. The goal was to assess whether it is possible to achieve $100 \%$ legibility of letters stored in the crate using a postal RFID technology.

To determine the success of reading measurements were performed on letter mail stored in the actual postal crate using the RFID reader and two antennas from Alien, label affixed to objects and middleware management program. Under review was to create RFID systems and perform test measurements to evaluate the success of the load of letters stored in crates and stored the measurements are properly presented and evaluated in the framework to create web application related to middleware program that is designed to manage RFID reader.

For the purposes of measurement was the technical background of Alien - RFID reader, RFID tags, and two antennas, which was created by the RFID gate. Used middleware program provided by the Italian company Aton, s.p.a. web application was developed in an environment with a PHP MySQL database system. Principle of RFID technology is as follows:

- the base of the system is reading device (reader) RFID systems and serves as a transmitter and a receiver of radio waves

- part of the reader are one or more antennas through which the reader is able to transmit electromagnetic waves to a radiofrequency, and transmit the encoded information,

- using RFID transponder tag is received electromagnetic waves with information encoded converted into an electric charge is stored on RFID tags,

- transformation of electromagnetic waves into an electrical charge is possible that the RFID tag is able to broadcast their own radio waves with its own unique encoded information,

- reader receives the signal modulated with disabilities. The information thus obtained is further processed and sent to the superior information systems.

\subsection{Orientation and location of identifier}

Identifiers are polarized as well as antennas. For optimal performance RFID read range and the polarization must be parallel to the polarization of the antenna. For most of the current is the polarization parallel to the longer side. Ideal antenna alignment and location identifier is an identifier in front of the antenna and the longer side oriented parallel to the polarization of the antenna. Real but it is virtually impossible to guarantee. In all applications, but it is important to align the antenna with the antenna system identifier reader. Same alignment orientation identifier in phase with the direct model antenna returns optimal results. However, the general rule is that the identifier may be disoriented by about $15^{\circ}$ angle in any direction with negligible performance degradation. Correct adjustment of the system may allow an even greater tolerance. This tolerance to disorientation system allows you to read the label orientation and angle of presentation changes depending on their trajectory through reading. 


\section{Reading}

Range

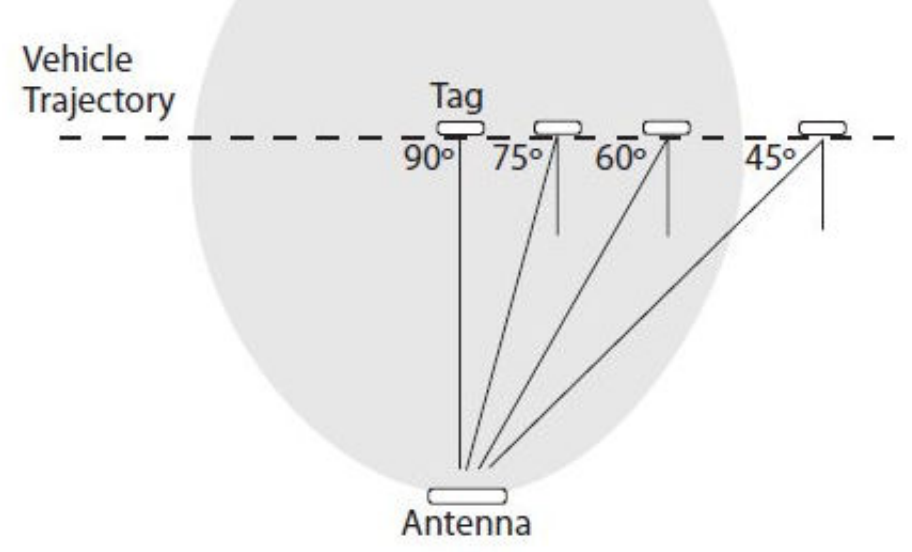

Figure 16. This is illustration shows the identifier transmitted by antennas around the reader.

This illustration shows the identifier transmitted by antennas around the reader. As shown in figure 17. range reading is weaker if the identifier to a greater angle to the antenna.

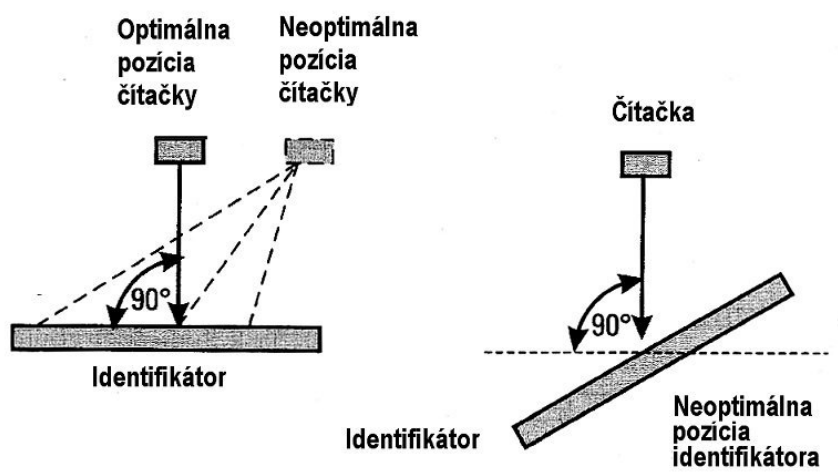

Figure 17. The optimal position of antenna and tag (identifier) 
Reading range may be affected by pitch, roll or diverting of identifier. In a further assume that the antenna polarization is parallel to the long side identifier.
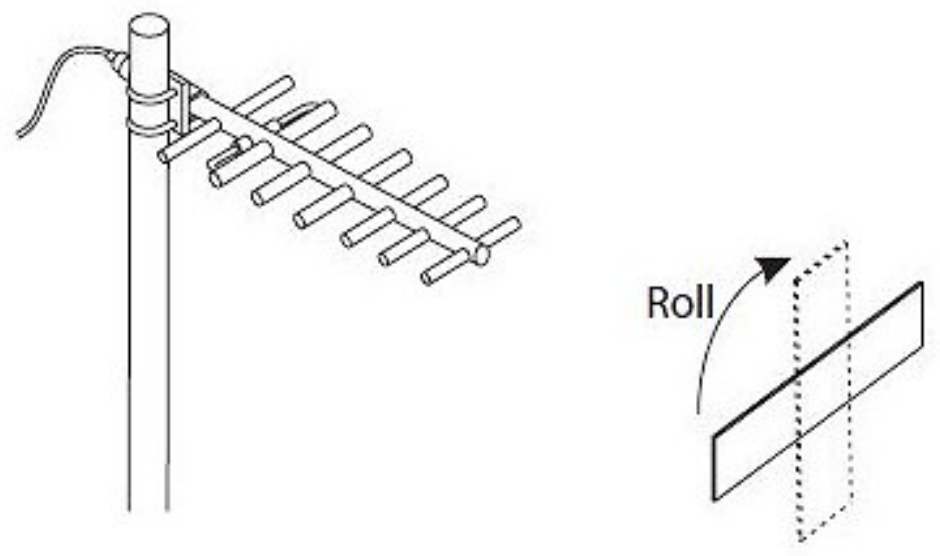

Scheme 1. Rotation (roll) the identifier in a clockwise or counterclockwise direction will cause loss of performance. This loss increases with expanding angle of rotation - the optimum approach angle is $90^{\circ}$. That may be why the orientation of the identifier used to avoid reading other remote signals from any recources.
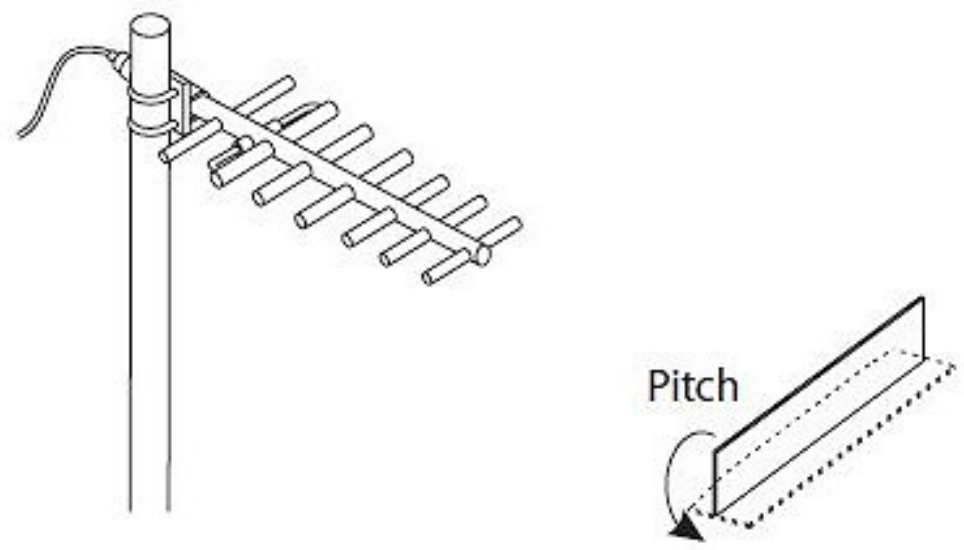

Scheme 2. Inclination or tilt (pitch) of identifier - the rear rotation (front to back) around a horizontal axis) affects performance only slightly. 

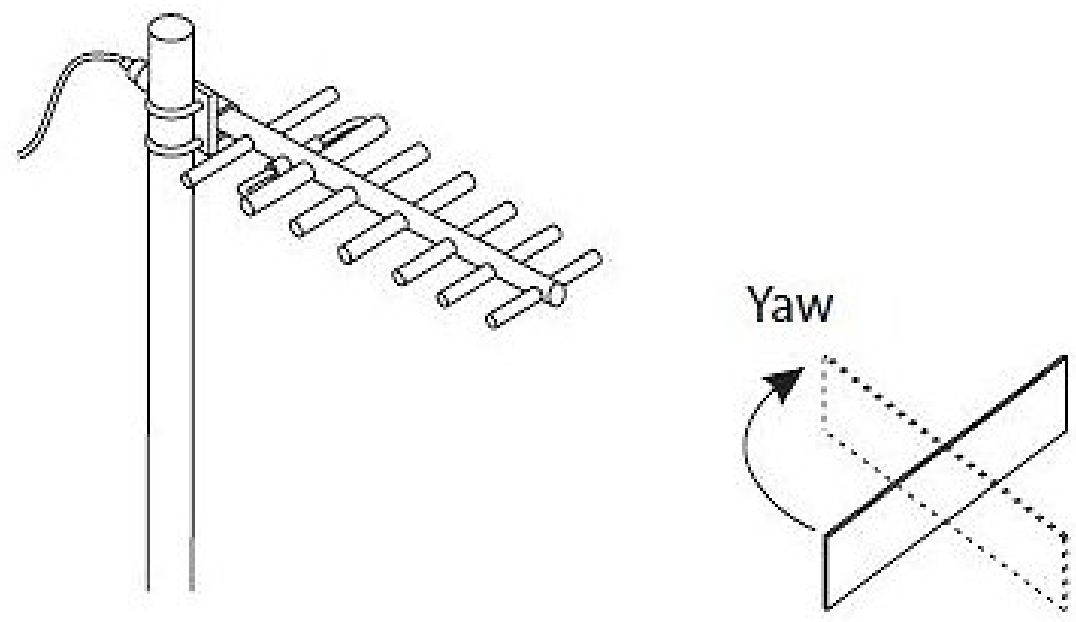

Scheme 3. Rotation (yaw) facing each other (end on end) about its vertical axis is for further consideration. As the angle of identifier rotation increases from the antenna, area identifier's internal antenna, which is within the reader field is shrinking. With this reduction is also associated identifier readability.

Differences in surface mounting, the angle and height of placement, as well as changes in the angle of the transition identifier reading area are compensated if allowed a sufficient margin for optimal orientation and alignment

\subsection{Evaluation of test success}

The main purpose of making measurements and the creation of applications was to evaluate the success of the measurements and draw conclusions about the most significant and important impacts that affect the success of reading. The effects have been studied for measurements are as follows:

- save correspondence with the measurements

- the use of crates,

- free storage correspondence,

- number of measured items.

\subsubsection{Impact of the imposition of letters}

During performing measurements correspondence can be letters stored in several ways to create a number of test locations. Basis to build positions in the imposition items vertically or horizontally as shown next figure. 
At horizontally aligned storage correspondence can be created as the following positions:

- items stored horizontally narrow side facing the antenna,

- items stored horizontally wider side facing the antenna,

- items stored horizontally, in the gate rotated 360 degrees.

At a vertical aligned correspondence can be created as the following positions:

- Items stored vertically, towards the flat antenna

- items stored vertically, perpendicular to the flat antenna

- items stored vertically in the gate rotated for about 360 degrees.

Other than those specified positions were also carried out tests on unaligned shipments. At vertical alignment shipment is possible to distinguish whether the letter is placed RFID tags glued upward or downward. Depicting the imposition of letters is shown in the following table. In order to determine the optimal storage correspondence tests were performed in all these positions.

\begin{tabular}{ll}
\hline Description deposit of letter items & Reading success (\%) \\
\hline Aligned vertically toward the surface of the antenna, the tags below & 29,03 \\
\hline Aligned vertically toward the surface of the antenna, the tags above & 64,98 \\
\hline Aligned vertically, perpendicular to the flat antenna, the tags below & 4,15 \\
\hline Aligned vertically, perpendicular to the flat antenna, the tags above & 11,98 \\
\hline Horizontally aligned, wider side facing the antenna & 26,27 \\
\hline Horizontally aligned, narrow side facing the antenna & 11,06 \\
\hline Aligned vertically, rotate the gate about 360 degrees & 65,44 \\
\hline Aligned horizontally, rotate the gate about 360 degrees & 42,40 \\
\hline Misaligned, randomly placed & 85,25 \\
\hline
\end{tabular}

Table 1. The impact of the imposition of letters

As shown in table above the highest percentage was reached at a loading unaligned accidentally saved letter. Saving is but random, and in greater numbers there is no guarantee that the RFID tags do not overlap more than one shipment. We can assume that for larger numbers, this percentage declined.

When comparing the measurements of success with storing correspondence RFID tags up and save measurements made with RFID tags can be seen down a significant difference. Greater success is achieved when depositing RFID tag upwards, which is due to greater freedom for the RFID tags. Large differences are visible when you turn the leaf surface ship- 
ments towards RFID tag antenna. Compared to the stored correspondence surface perpendicular to the RFID tag antenna is the difference in the success of loading more than $50 \%$.

Measurements were carried out with the type of gate in which both antennas are on the sides. Gate type significantly influenced the success of horizontal loading and shipments compared to a vertical were significantly lower. Rotate the trays in the gate 360 degrees slightly increased readability vertically or horizontally stored correspondence. Optimal solution in terms of deposit of letters on the measurements is to store:

- vertical,

- flat plate toward the RFID antenna,

- Implementation in gate turned 360 degrees.

\subsubsection{The impact of the use of containers}

To determine the impact of the imposition of letters in the crate measurements were not made only in the crate, but also in bulk correspondence without containers. With the settings and save the items in the same position was achieved the following results:

\begin{tabular}{lcc}
\hline & Reading success (\%) \\
\hline Imposition of letter items & use crate & without crate \\
\hline aligned, horizontally placed & 82,95 & 84,79 \\
\hline aligned, vertically, tag surface to side antenna & 91,71 & 63,59 \\
\hline aligned, vertically, tag surface upright to side antenna & 53,46 & 46,08 \\
\hline aligned, vertical rotation & 90,32 & 80,18 \\
\hline aligned, horizontally rotation & 83,41 & 93,09 \\
\hline unaligned, random stored & 98,16 & 96,31 \\
\hline overall & 83,35 & 77,34 \\
\hline
\end{tabular}

Table 2. The impact of the use of containers

The table shows that the use of containers has not a significant impact on the success of improvement or deterioration reading of letter items. When using containers to store the correspondence is achieved even greater average success on reading. This is probably due to the freer depositing correspondence in containers than in the same position simulations without containers, especially in the upright position. At horizontal position, where it was easy to simulate the same position was achieve slightly higher readability without the use of crates. 


\subsubsection{The impact of free scope of stored letter items}

By examining the different variations of the deposit of letter mail has proved an important factor affecting the success of slackness between RFID tags glued to the letter. It was had done testing with the following settings of slackness between the letter post:

- separate correspondence by carton;

- the bulk correspondence (classical)

- letter correspondence pressed together.

\begin{tabular}{ll}
\hline Degree of freedom between the letter items & Successful reading (\%) \\
\hline pressed together & 68,66 \\
\hline stored slackness & 69,59 \\
\hline separate by carton & 100,00 \\
\hline
\end{tabular}

Table 3. The impact of free scope of stored letter items

The table shows that the separation of the carton shipments has a significant impact on the success of loading achieved is $100 \%$ readable. Crushed shipments only slightly worse compared to the readability of bulk shipments.

\subsubsection{The impact of other elements}

Unit shipments for the cardboard several measurements were carried out to confirm $100 \%$ readability:

\begin{tabular}{lrr}
\hline Studied impact & \multicolumn{2}{c}{ Successful reading (\%) } \\
\hline speed of running by gate & $4 \mathrm{sec}$ & 100,00 \\
\cline { 2 - 3 } & $2 \mathrm{sec}$ & 100,00 \\
\cline { 2 - 3 } antenna distance & quickly & 100,00 \\
\cline { 2 - 3 } & $80 \mathrm{~cm}$ & 100,00 \\
\hline antenna intensity & $60 \mathrm{~cm}$ & 100,00 \\
\cline { 2 - 3 } & $90 \%$ & 100,00 \\
\cline { 2 - 3 } & $75 \%$ & 100,00 \\
\cline { 2 - 3 } & $60 \%$ & 100,00 \\
\cline { 2 - 3 } & $40 \%$ & 100,00 \\
\hline optimal storage of letter & $20 \%$ & 95,85 \\
\hline
\end{tabular}

Table 4. The impact of other elements 
By separating mail boxes are reaching nearly all settings by $100 \%$ readable. Mild impairment occurred only at very low intensity at $20 \%$ and save correspondence area perpendicular to the antenna. But even in these cases is very high loading percentage. But the question remains questionable real use in practice.

\subsection{Model 1 - Evaluation of the feasibility of using RFID technology}

At real-saving correspondence to crates are stored in the manner:

- aligned vertically,

- unseparated to each other as shown next figure.

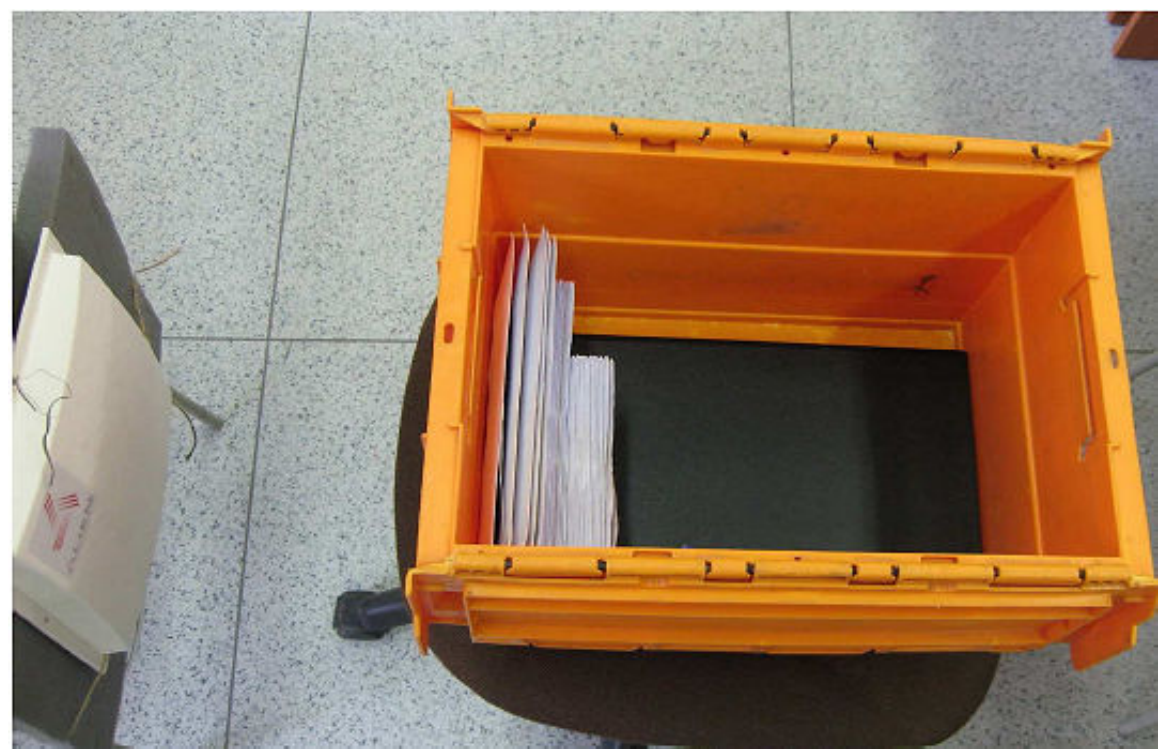

Figure 18. Postal crate

By a given type of deposit correspondence, the maximum load value of the success achieved in the following settings:

- intensity of the RFID reader to a maximum value,

- type of gate - one antenna on the side, a top antenna,

- remain in the gate at least 2 seconds,

- freely save letter items.

After several performed in those settings with 31 letter items were obtained the following results: 


\begin{tabular}{lll}
\hline Measurement count & Number of reading items & Successful reading (\%) \\
\hline 1 & 30 & 96,77 \\
\hline 2 & 29 & 93,55 \\
\hline 4 & 30 & 96,77 \\
\hline 5 & 30 & 96,77 \\
\hline 6 & 24 & 77,42 \\
\hline 7 & 25 & 80,65 \\
\hline Overall & 31 & 100,00 \\
\hline
\end{tabular}

Table 5. Results of measurement

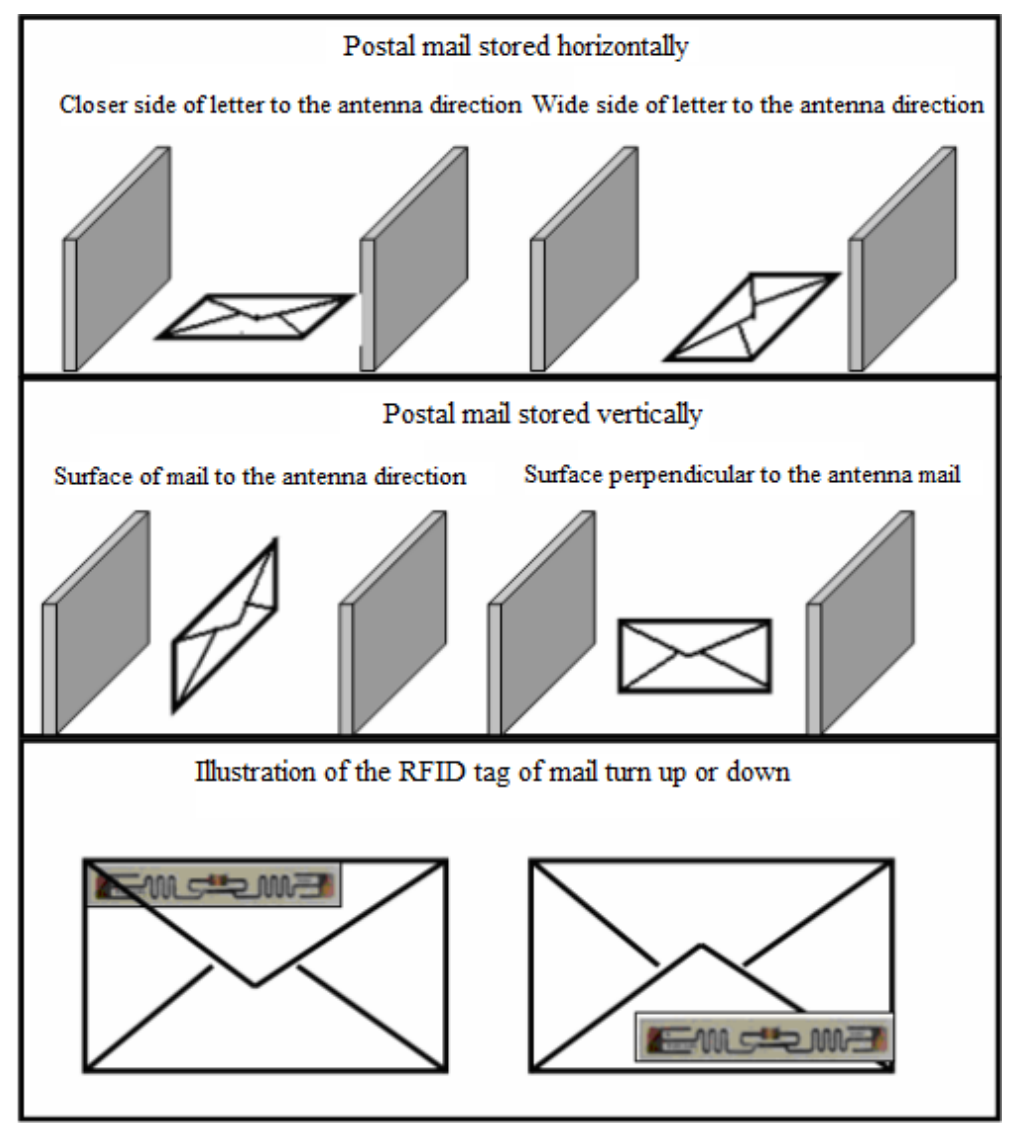

Figure 19. Position of mail while reading 
At horizontally stored correspondence, following positions can be created:

- items stored horizontally narrow side facing the antenna,

- items stored horizontally wider side facing the antenna,

- items stored horizontally, in the gate rotated in 360 degrees.

At a vertically stored correspondence, following positions can be created:

- items stored vertically, surface towards the antenna

- items stored vertically, surface perpendicular to the antenna

- items stored vertically in the gate rotated in 360 degrees.

Except for those specified positions, tests were also realized on unaligned shipments. Within vertically aligned shipment, it is possible to distinguish whether an RFID tag is placed upward or downward on letter. Depicting the imposition of letters is shown in the following table.

In order to determine the optimal storage of correspondence, the tests were performed in all mentioned positions.

\begin{tabular}{ll}
\hline Description deposit of letter items & Success of reading (\%) \\
\hline Aligned vertically toward the surface of the antenna, tags placed on bottom & 29,03 \\
\hline Aligned vertically toward the surface of the antenna, tags placed on top & 64,98 \\
\hline Aligned vertically, perpendicular to the flat antenna, tags placed on bottom & 4,15 \\
\hline Aligned vertically, perpendicular to the flat antenna, tags placed on top & 11,98 \\
\hline Horizontally aligned, wider side facing the antenna & 26,27 \\
\hline Horizontally aligned, narrow side facing the antenna & 11,06 \\
\hline Aligned vertically, rotate the gate about 360 degrees & 65,44 \\
\hline Aligned horizontally, rotate the gate about 360 degrees & 42,40 \\
\hline Misaligned, randomly placed & 85,25 \\
\hline
\end{tabular}

Table 6. The success of reading the different position of mail

\subsection{Model 2 - Test of readability of postal items through the RFID technology}

One of the methods that could significantly make the process of identifying postal items in transport condition more effective is above mentioned RFID technology. As a wireless technology, without visual contact with the shipment, it tracks and identifies the contents without the need of manual handling from the crate. This allows easier and more efficient handling of supporting documents (creating the list of items, checking the presence of item) of postal sacks/bags and containers. With regard to price and the quantity of items processes, a question arrives: Is RFID technology effective and should be used for all shipment, including letters? As already mentioned - due to the large quantities of letter items and still quite high price of RFID tags - the method could be appropriate only for registered letters/mail. The actual implementation design of RFID technology, as shown in Figure 3 could be divided into the following phases: 
1st phase - tracking between the HSS

2nd phase - tracking between the HSS and OSS.

3rd phase - tracking between the OSS and final post office (point of delivery).

Because of our basic interest is in the RFID technology we tried to test of readability RFID tags placed on postal items in various situations. Basic assumption is the use of RFID gates at the entrance and output to the processing unit as show next figures.
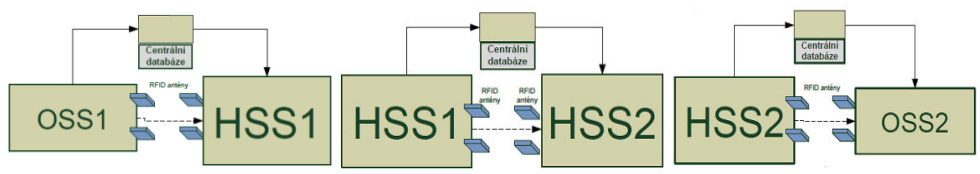

Figure 20. RFID gates at the entrance and output to the processing unit

In order to verify the practical applicability of this technology we have dealt with the preparation and implementation of practical activities through which we examined reading RFID tags. The object of these measurements was to determine the statistical characteristics of reading success and reading passive tags, placed on postal items, located in the mail bag. The aim was to provide sufficient information as accurately measured under different conditions that can occur in a real situation, including a draft measure, which would lead to the improvement of measured data.

Therefore we try to simulate a real postal process in conditions close to operational and test this technology on next component set configuration:

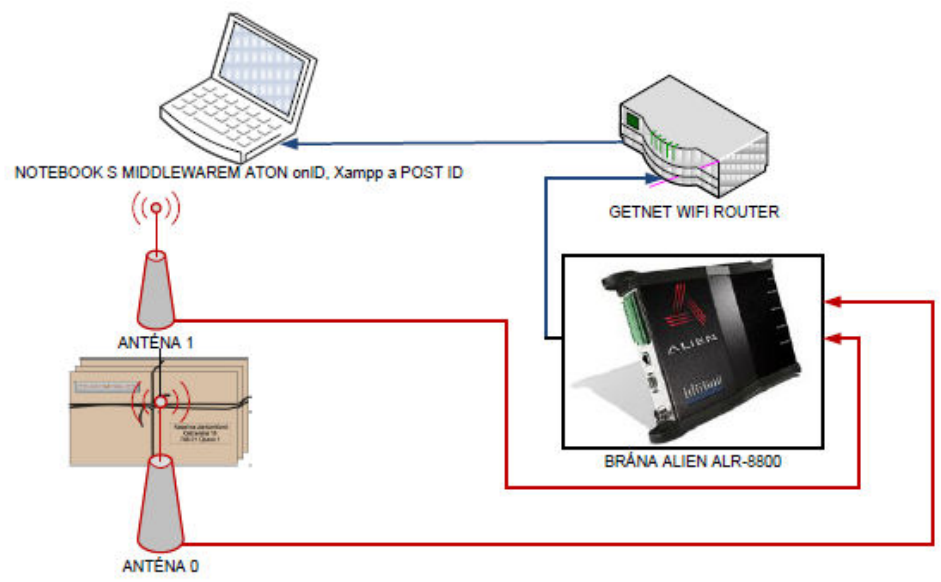

Figure 21. Principle component links 
When some bundle or bundles placed in a bag entry into the detection field begin to transfer the identification data from the RFID tag to the antennas of gate. The gate extends the data (by adding date, time, number of particular antenna,...) and send them to system. Thus processed data are transmitted through the wifi router middleware Aton onId into notebook.

Figure 21 (figure located in next section) describes the principle of software components and their cooperation - communication between web applications POST ID, MySQL database server and middleware onid Aton.

\subsubsection{Description of the model}

There was used a software from Italian company Aton, also known as middleware, which provides the management, organizational and communication operations between different applications. In our case, the firmware Alien Gate and other applications, particularly database server. Onid Aton itself is not monolithic program, but it is a functional connection Java service console (java server) and the graphic manager called Qflow. Itself Qflow intuitive and easy enabled an interactive creation and administration of custom processes.

Major elements are program elements, called the processor to implement elementary operations (reading from the gateway, filtering, record the output, etc..)

- The first step is to enter the configuration data to POST ID. From there shall be deposited directly into database tables. The subject of this storage is data on the number of configuration items and numbers.

- In a second step, after the start of broadcasting alien element and their detection by InlineProcesor made load measurement numbers, the number of items and the configuration number and attach it to information from the antennas.

- In third step, the data are extended by the information about time and date using TimeFormatter processors. The first two into generators of text and xml files with a resolution by the uniqueness of the registration data. The third way into InsertProcessor, where the data are entered into the database. Fourth way turns itself to LackEvents processor. In the case that in a defined time there is here not recorded any new message from the gateway, it sends a new message to next two processors, which on the basis of the received message (MessageGenerator) to increase the value of measurement number by 1 and this value by updating the database InsertProcessorA.

- The second CommandExecutor processor on receipt of a report by running the alarm indicates the new number of measurements. The measurement consists of setting values in POST ID and physical adjustment of antennas. The effort was to make sure if it was possible the most accurate and smoothest possible transition from the beginning to the end of the runway. After making the transition waiting for the time needed for detection of zero, which means the CPU and LackEvents CommandExecutor will sound, indicating the end of measurement and readiness for the next measurement. At the same time processor MessageCounter increased number of first measurement after finishing the sound detection is possible again to make the switch between the antennas to the selected track. 
Full application part is shown in Figure 22.

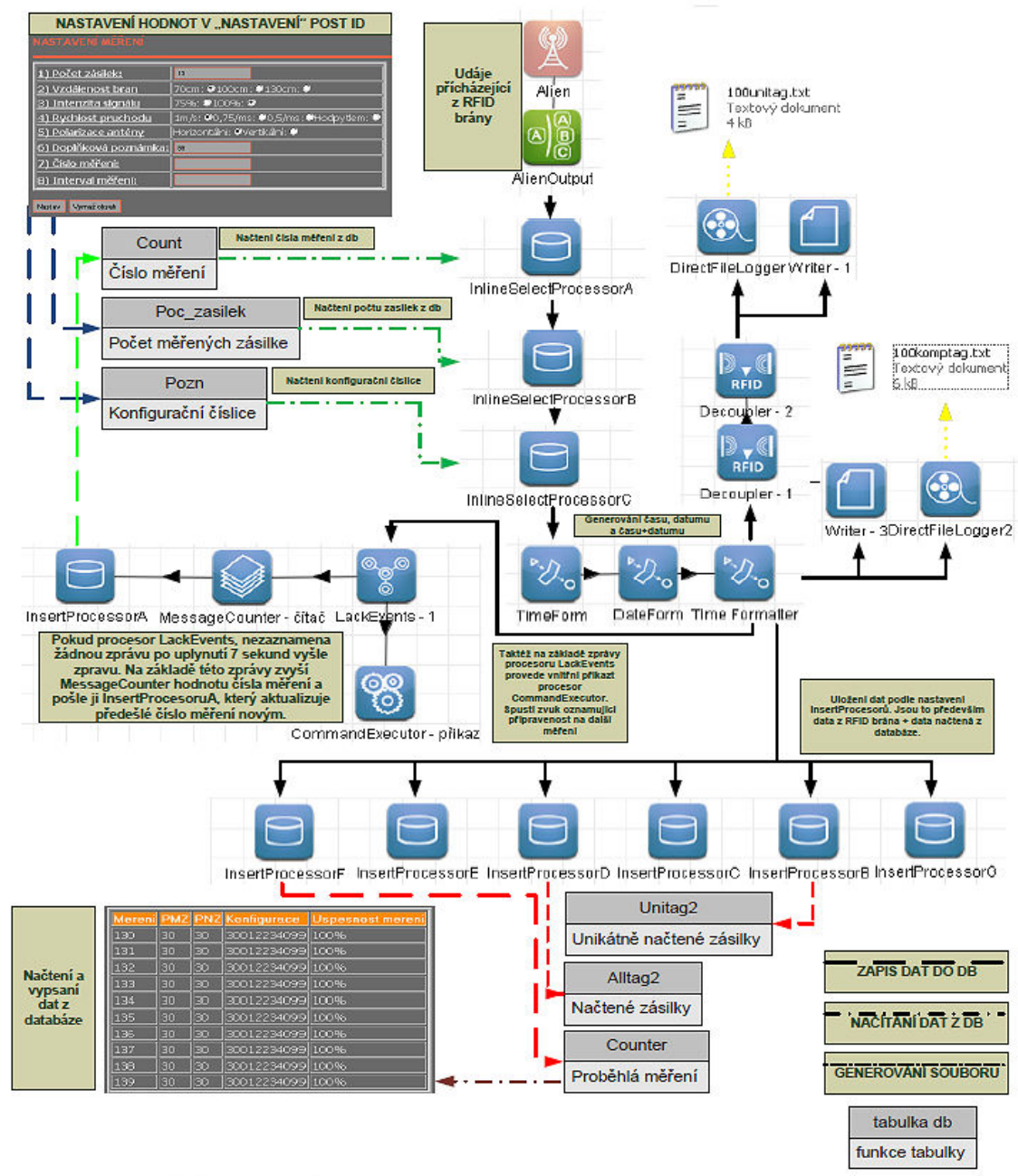

Figure 22. Final configuration model based on ATON onID 


\subsubsection{Test of readability of postal items}

Measurements carried out in an improvised laboratory in the premises of the computer lab of the University of Žilina. There were measured passive tags placed uniformly on all mail in the middle of the upper left corner. Tags were placed so strictly because simulate challenging situation that could occur in real practice, so that all shipments under the labels overlap, the close neighbours. This arrangement could cause the EM waves emitted by RFID tags will interfere with each other. For each item was then transcribed RFID tag number and serial number marked for later processing easier statistical information. The object of measurement items were deposited into the mail bags, which are grouped into a bundle. To determine the characteristics of reading and expanding sub-measure was introduced by another character, and that is the position of the beam due to the antennas. These positions are defined (according to Figure 23):

1. boundle horizontally - the length of the area enclosing antennas,

2. boundle horizontally - the width of the area enclosing antennas,

3. boundle vertically - party address shipments parallel flat antennas,

4. boundle vertically - mail address side perpendicular to the plane antenna.

Likewise, in our measurements were sequenced according to the serial number of items, grouped into bundles, according to the size of the consignments as shown in Figure 23.
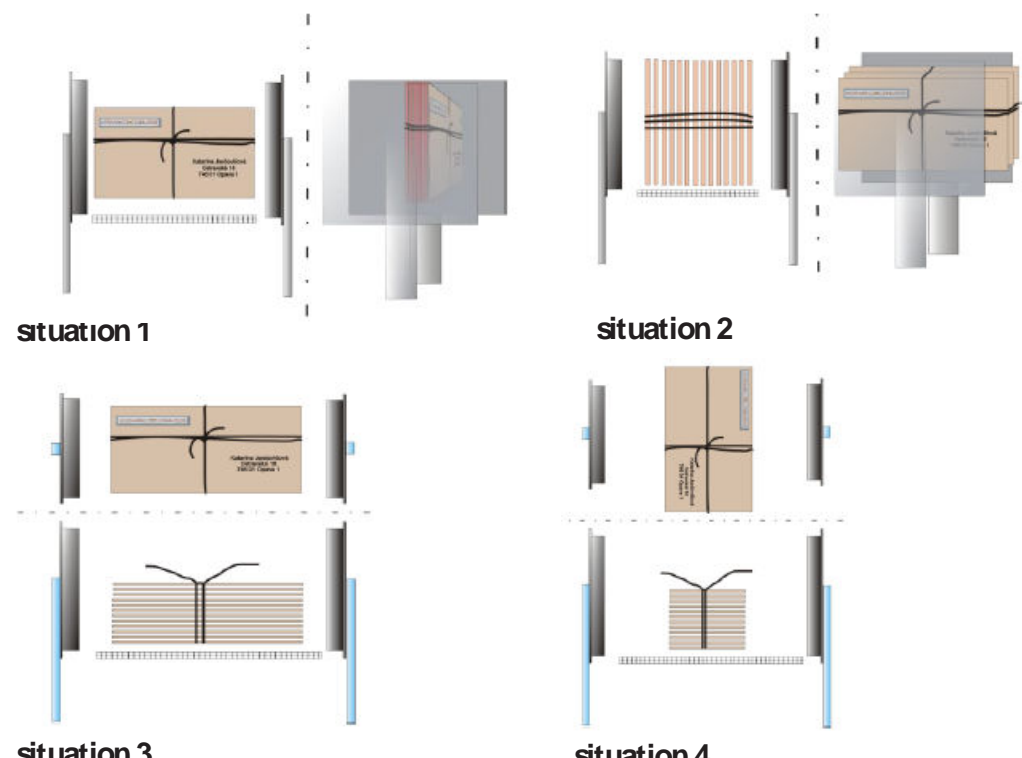

situation 4

Figure 23. Configurations of letters bundles 


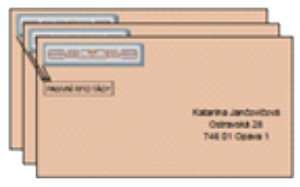

Set $A$

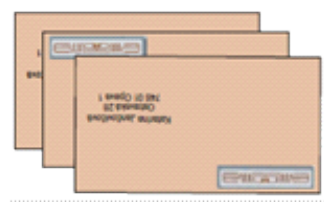

Set $B$

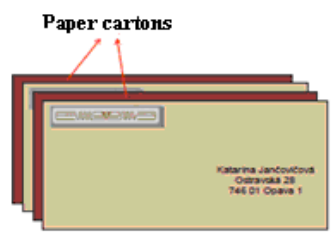

Set $C$

Figure 24. Several set of letter bundles

We have used two ways of transporting items via gate:

1. Static transfer through the postal truck or conveyor, also examined the transport unit volumes, which are in relative peace in terms of positioning items

2. Dynamic hand, respectively manual transfer using human power - move with a rate shocks, which could help to better read the labels in bundles.

All data recorded after the measurement time was subject of evaluation, and because of the large scale of the recorded data was be evaluated only average and cumulative results Determining the accuracy of measurements based on the statistical characteristics - it is a statistical description, which expresses the degree of statistical variability of the file, it indicates the letter R, It indicates the difference between the largest and smallest value and in some extent we are able to denounce both the large inaccuracies in the measurement occurred. It is expressed by the formula $R=X_{\max }-X_{\min }$. In percentage terms inaccuracies modified formula looks as follows:

$Z=\left(\left(X_{\max }-X_{\min }\right) /\right.$ number_of_items $) * 100$

Based on this formula was compiled by chart positions inaccuracies sets.

Since the evaluation of this quantity of data with the graphic processing is substantially opaque (a sample can be figure 25 with a graphical evaluation, which is a preview of kits depending on the speed of transition between the antennas) and it is not possible to present all the results of measurements on such a small space of this contribution will sum up only the basic results of the measurements and focus only on some important findings.

The measurement is made clear that that some parameters are irrelevant in terms of readability, such as speed of shipments run through the transition zone readers are relatively independent (readability in an average of about $76 \%$ to $2 \%$ deviation, the readers distance is taken with a $77 \%$ deviation around $6 \%$ use conveyor with $80 \%$ deviation around $4 \%$, or manual switch $(94 \%$ with a deviation of about $2 \%$.

It is interesting that in an evaluation of readability situations of the consignment given the readers runs through the gate (table 1 upper), in some cases is sufficient and relatively uniform (situation 2 and 3 value of 100\%), while what for example situation 4 is the readership in wide range of $2 \%$ up $92 \%$. 


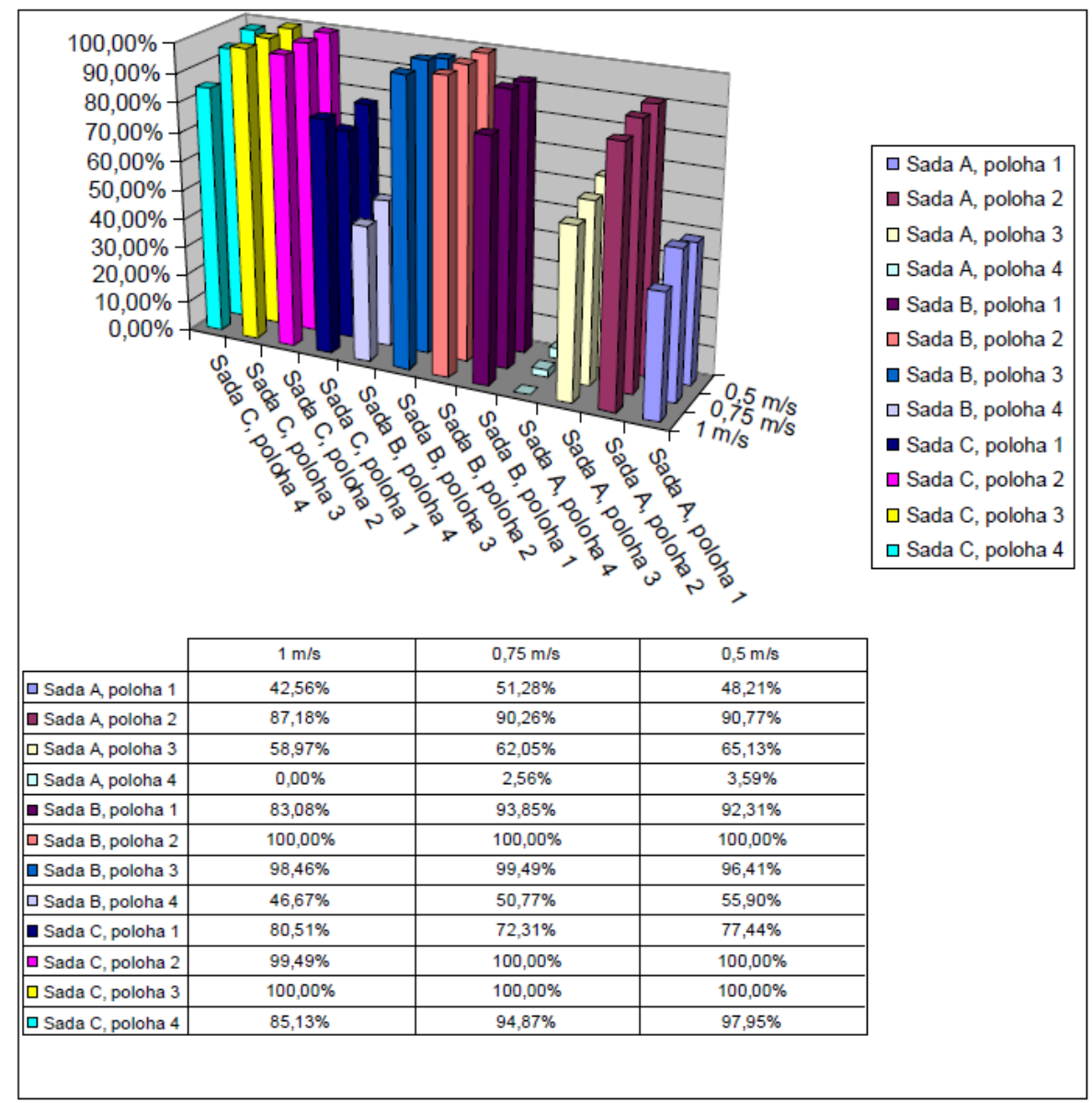

Figure 25. Final test results

\begin{tabular}{rrrrrr}
\hline Set & situation 1 & situation 2 & situation 3 & situation 4 & average \\
\hline $\mathrm{A}$ & $47,35 \%$ & $89,40 \%$ & $62,05 \%$ & $2,05 \%$ & $50,2 \%$ \\
\hline $\mathrm{B}$ & $89,74 \%$ & $\mathbf{1 0 0 , 0 0 \%}$ & $98,12 \%$ & $51,11 \%$ & $84,7 \%$ \\
\hline $\mathrm{C}$ & $76,75 \%$ & $99,83 \%$ & $\mathbf{1 0 0 , 0 0 \%}$ & $92,65 \%$ & $92,3 \%$ \\
\hline
\end{tabular}

Table 7. Basic dependency between sets of readability and situations

The overall success of the method of transition as the distance of antennas for different speed ranges from $81 \%-87 \%$, and has a major impact on readability, similar to the way the transition between sets of antennas is relative stable (81\% to $95 \%$ ) 
Based on the evaluation of measurement data cannot be identified unambiguously exclude or recommend the use of this technology in practice. These measurements may be partly conditional on imprecision caused by a provisional Laboratories. There is unable to clearly provide the desired stable speed and position of shipments due to the antenna. The end result is therefore a lack of readability of RFID tags in a traditional way-now commonly used in practice in the post measurement known as the set A. Although in other cases, the readability is very high and almost $100 \%$ (set C or B), there were other aspects that significantly affect its use.

\section{Conclusion}

RFID technology is still growing up and there is several type of application, which you can use in condition of the postal processes. This chapter deals with type of application that are common uses in postal sector such as mail bag, letter trays, roll cages and vehicle tracking and managing application. All these application are useful for the track and trace system and it presents added value for costumers. Most postal services provide at least a limited form of track and trace, particularly for premium delivery services. Today, tracking uses bar codes. Switching to RFID tags can lower tracking labor by eliminating the need for most manual piece handling. RFID is a very useful and exciting technology. It seems that everywhere one looks there is some article about RFID and the huge benefits its technology promises. Moreover, there are many examples that demonstrate how this technology is fulfilling its potential.

Based on the measurements it can be concluded, with some exceptions that prove the rule, the closer they are to each antenna, the greater the success of reading RFID tags. Given the large dispersion of values it can be concluded that some elements are simply eliminated They can, for example using multiple counting gates, respectively antennas (eliminating the position of shipments), or the use of such specialty (bubble) envelopes for magnification air gap between consignments (as by set $\mathrm{C}$ )

This article deals with identification of postal items and transport units in logistic chain of postal operators. It described scheme of the transport process, including planned technology and there is also simulated a real postal process in conditions close to operational. Article is part of the projects described below, which, together with the afore-mentioned application, will improve the learning process at the Department of Communications.

The benefits of RFID technology can be reaped if RFID events give realtime visibility to the business processes either already in place or to new ones. The backend systems give a business context to the RFID events collected from the RFID data collection tools and then invoke the right business process in real time (or near real time). Protecting the backend system is vital from the various security threats at the network level (attacking ONS or network communication between data collection tool and backend system) or at the data level (spurious events).The network level attacks can be prevented by using secured communications between various processes. The data attacks are hard to deal with, and application de- 
signers must take special care to differentiate spurious events from good events and then act on the good ones almost in real time. Since data is collected using automated data collection techniques, application designers must clean the repository where good RFID events are stored.

Costs of the security regarding RFID technology got implemented in a company's infrastructure still presents relatively expensive attribute in the eye of CEO's. Although the price of active and passive tags is still being reduced and RF technology becomes continuously more and more popular in a field of logistics, supply chains, toll systems, postal services, retailers or asset management, the relevance has to be put on a confrontation of costs of RFID implementation and its explicit use towards the eventual probability of attack.

In the relation on main aim of this article was focus on options of implementation mobile technology includes RFID technology into postal transport area. The content of introductory chapter is approximation the best terms of category and theoretical knowledge, which is used in this article. Further there are characterized the postal transport network, individual transport units, which is use in this area.

By optimal settings in real conditions the average maximum reading percentage is about $91.71 \%$. Significant effect to increase the readability, RFID tags have been pointing toward a flat antenna and the antenna by sensing the top. Very significant impact on increasing or reducing the readability of the number of letters had the crate. For the purposes of measurement was used in 31 letters. The real use of the crate contained a much higher number of letters, which would likely significantly reduce readability.

Based on these results, identification of letters in the crate, RFID technology is not yet, given the technical conditions for real. Achievements, however, were relatively high. Some uncertainties should be possible to eliminate appropriate technical configuration (number of gates and Antennas, their location, etc.). On the other side some, particularly the operating elements (separation of cardboard) can be solved as special packaging elements (bubble envelope to increase the air gap), etc.) can ensure a desired level of reliability required reading. Therefore, we expect that further testing with a larger number of antennas other types of antennas, readers and RFID tags in our AIDC laboratory.

\section{Acknowledgement}

This work was supported in part by grant and research project VEGA 1/0421/12 - Modeling diffusion of knowledge in business value chains (60\%), Centre of excellence for systems and services of intelligent transport II., ITMS 26220120050 supported by the Research \& Development Operational Programme funded by the ERDF $(20 \%)$ and institutional projects: 3/KS/2012 - Supporting education through educational multimedia applications [10\%] and 1/KS/2012 - Sensitivity analysis of contact points to the costs arising from the provision of UPS [10\%] 


\section{Author details}

Juraj Vaculík, Peter Kolarovszki and Jiří Tengler

*Address all correspondence to: juvac@fpedas.uniza.sk

University of Zilina, Faculty of Operation and Economics of Transport and Communications, Department of Communications, Slovakia

\section{References}

[1] Hunt V. D., Puglia a. , Puglia m., RFID: A Guide to Radio Frequency Identification, ISBN-13: 978-04701072007.

[2] Jones, ERIC, Chung, CHRISTOPHER : RFID in Logistics: A Practical Introduction, ISBN 978-0849385261, 2007

[3] Finkenzeller, K.: Fundamentals and Applications in Contactless Smart Cards and Identification. In: RFID Handbook. John Wiley \& Sons, 2006. ISBN 0-470-84402-7.

[4] LEHPAMER, H.: RFID Design Principles. Artech House, Inc, Norwood, MA, USA, 2008. ISBN 978-1-59693-194-7.

[5] Myerson Judith M., RFID in the Supply Chain: A Guide to Selection and Implementation, Syngres, 2007. 215 s. ISBN 978-08493301086.

[6] Poirier Charles, RFID Strategic Implementation and ROI : A Practical Roadmap to Success, J. Ross Publishing, Inc., 2006. 243 s. ISBN 1-932159-47-9.

[7] Švadlenka L. a kol. Transportation and communication system, První vydání. Pardubice : Univerzita Pardubice, 2006. 136 s. ISBN 80 - $7194-911-6$

[8] ŠVADLENKA, Libor. RFID in postal and courier services. In PRASAD, B. V. S. , KALAI , Selvan. Supply chain management in services industry : an introduction. 1st edition. Hyderabad (India) : Icfai Books, 2007. s. 68-74. ISBN 81-314-0756-X.

[9] Thornton F.,Haines B., Bhargava H., RFID Security, ISBN 1-59749-047-4, 2005.

[10] THORNTON, F. a kol.: RFID Security, Rockland: Syngres Publishing 2006. ISBN 1-59749-047-4.

[11] Zelik P.: Mobile technology in postal transport, University of Žilina, Faculty of economical and operation of $\mathrm{f}$ transport and communication, dissertation work

[12] Post Norway-Case Story. Located on: http://www.lyngsoesystems.com/Downloads/ 074.342.885-A4-Post-Norway-Case-Story.pdf, 2008

[13] Post Denmark - Case study. Located on: http://www.lyngsoesystems.com/Downloads/074.719.731-Post-DK-Case-Story-.pdf, 2008 
[14] Quality monitoring solution, Located on : http://www.lyngsoesystems.com/postal/ quality_monitoring.asp, 2008

[15] Canada Post case story. 2008. [online]. Lyngsoe Systems (Canada). [citované 2009-03-15]. Dostupné na: http://www.lyngsoesystems.com/ Downloads/074.772.592Canada-Post-casestory-A4.pdf

[16] Garfinkel, S - Rosenberg, B, RFID Applications, Security, and Privacy, Addison- Wesley Professional, 2005.

[17] DENNIS E. BROWN. RFID Implementation. New York (USA), McGraw-Hill Publishing, 2007

[18] Christina Soh, Sia Siew Kien, and Joanne Tay-Yap, Enterprise resource planning: cultural fits and misfits: Is ERP a universal solution? Communications of ACM, 43, 4751, 2000.

[19] Benita M. Beamon, Supply chain design and analysis: Models and methods, International Journal of Production Economics, 55, 281-294, 1998.

[20] D.M. Lambert and M.C. Cooper, Issues in supply chain management, Industrial Marketing Management, 29, 65-83, 2000.

[21] Valerie T., Neckel W., Information technology and product lifecycle management, In Proceeding of the 1999 IEEE International Symposium on Electronics and the Environment. 